\title{
ORTHOGONAL SPLINE COLLOCATION \\ LAPLACE-MODIFIED AND ALTERNATING-DIRECTION METHODS FOR PARABOLIC PROBLEMS ON RECTANGLES
}

\author{
BERNARD BIALECKI AND RYAN I. FERNANDES
}

\begin{abstract}
A complete stability and convergence analysis is given for two- and three-level, piecewise Hermite bicubic orthogonal spline collocation, Laplacemodified and alternating-direction schemes for the approximate solution of linear parabolic problems on rectangles. It is shown that the schemes are unconditionally stable and of optimal-order accuracy in space and time.
\end{abstract}

\section{INTRODUCTION}

In this paper, we present and analyze several two- and three-level schemes for the approximate solution of the parabolic problem

$$
\frac{\partial u}{\partial t}+L u=f(x, y, t), \quad(x, y, t) \in Q \equiv \Omega \times(0, T],
$$

$$
\begin{aligned}
& u(x, y, 0)=g_{1}(x, y), \quad(x, y) \in \Omega, \\
& u(x, y, t)=g_{2}(x, y, t), \quad(x, y, t) \in \partial \Omega \times(0, T],
\end{aligned}
$$

where $\Omega=(0,1) \times(0,1), \partial \Omega$ denotes the boundary of $\Omega$, and the linear differential operator $L$ is given by

$$
\begin{aligned}
L u= & -\frac{\partial}{\partial x}\left(a_{1}(x, y, t) \frac{\partial u}{\partial x}\right)-\frac{\partial}{\partial y}\left(a_{2}(x, y, t) \frac{\partial u}{\partial y}\right) \\
& +b_{1}(x, y, t) \frac{\partial u}{\partial x}+b_{2}(x, y, t) \frac{\partial u}{\partial y}+c(x, y, t) u .
\end{aligned}
$$

Orthogonal spline collocation with piecewise Hermite bicubics is used for the spatial discretization. Perturbations of the Euler method and the trapezoidal rule are employed for the time discretizations to produce Laplace-modified (LM) and alternating-direction implicit (ADI) schemes. We show that the LM and ADI schemes are unconditionally stable with respect to the spatial and time discretization stepsizes and that they are of optimal-order accuracy in the $H^{1}$ and discrete maximum norms for the space and time variables, respectively.

Received by the editor April 14, 1992.

1991 Mathematics Subject Classification. Primary 65M12, 65M60, 65M70.

(C) 1993 American Mathematical Society $0025-5718 / 93 \$ 1.00+\$ .25$ per page 
Finite element Galerkin LM and ADI methods for solving parabolic problems in two space variables were analyzed in $[15,18]$. Mimicking these methods, several authors have formulated and implemented some orthogonal spline collocation LM and ADI schemes and demonstrated experimentally their stability and convergence properties; see, for example, $[1,6,7,8,9,10,11,12,13,22,23]$. However, no theoretical convergence analysis has been given for any of these schemes. It should be noted that orthogonal spline collocation for parabolic problems in one space variable was analyzed in [19]; see also [14]. Recently, in [21] and [20], optimal a priori $L^{2}$ - and $H^{1}$-error estimates were derived for ADI collocation methods applied to the inhomogeneous heat and wave equations, and to separable parabolic and second-order hyperbolic problems. In our convergence analysis of orthogonal spline collocation for parabolic problems in two space variables, we follow the approach of [2] for analyzing orthogonal spline collocation for elliptic problems. This approach is based on using the piecewise Hermite bicubic interpolant of the exact solution as a comparison function.

In this paper, we present only theoretical analyses of two-level and three-level piecewise Hermite bicubic orthogonal spline collocation LM and ADI schemes for solving linear parabolic problems. In a companion paper [4], we discuss implementations of these schemes and present results of numerical experiments. It should be pointed out that at each time level, the LM methods require the solution of elliptic orthogonal spline collocation problems corresponding to Poisson's equation. Such problems can be solved efficiently by the recently developed fast Fourier transform direct algorithm of [3]. On the other hand, the ADI methods involve the solution of independent sets of one-dimensional orthogonal spline collocation two-point boundary value problems. Such problems give rise to so-called almost block diagonal linear systems that can be solved by the package COLROW [16, 17].

A brief outline of this paper is as follows. Preliminaries and general stabilty theorems for two- and three-level schemes in Hilbert spaces are given in $\S 2$. Two-level LM Euler and ADI Euler methods and the ADI Crank-Nicolson orthogonal spline collocation scheme are analyzed in §3. Three-level LM and ADI schemes which are the counterparts of the LM and ADI finite element Galerkin methods of $[15,18]$ are analyzed in $\S 4$.

\section{Preliminaries}

2.1. Partitions, piecewise polynomial spaces, Gauss points. Let $\left\{x_{k}\right\}_{k=0}^{N_{x}}$ and $\left\{y_{l}\right\}_{l=0}^{N_{y}}$ be two partitions of $[0,1]$ such that

$$
x_{0}=0<x_{1}<\cdots<x_{N_{x}-1}<x_{N_{x}}=1, \quad y_{0}=0<y_{1}<\cdots<y_{N_{y}-1}<y_{N_{y}}=1 .
$$

Let $h_{k}^{x}=x_{k}-x_{k-1}, h_{l}^{y}=y_{l}-y_{l-1}$, and let

$$
\begin{gathered}
\underline{h}_{x}=\min _{k} h_{k}^{x}, \quad \bar{h}_{x}=\max _{k} h_{k}^{x}, \quad \underline{h}_{y}=\min _{l} h_{l}^{y}, \quad \bar{h}_{y}=\max _{l} h_{l}^{y}, \\
h=\max \left(\bar{h}_{x}, \bar{h}_{y}\right) .
\end{gathered}
$$


It is assumed that the collection of the partitions $\left\{x_{k}\right\}_{k=0}^{N_{x}} \times\left\{y_{l}\right\}_{l=0}^{N_{y}}$ of $\Omega$ is regular, that is, there exist positive constants $\sigma_{1}, \sigma_{2}$, and $\sigma_{3}$ such that

$$
\sigma_{1} \bar{h}_{x} \leq \underline{h}_{x}, \quad \sigma_{1} \bar{h}_{y} \leq \underline{h}_{y}, \quad \sigma_{2} \leq \frac{\bar{h}_{x}}{\bar{h}_{y}} \leq \sigma_{3} .
$$

Throughout the paper, $C$ denotes a generic positive constant which may depend on $\sigma_{1}, \sigma_{2}$, and $\sigma_{3}$.

Let $\mathscr{M}_{x}$ and $\mathscr{M}_{y}$ be spaces of piecewise Hermite cubics defined by

$$
\begin{aligned}
\mathscr{M}_{x} & =\left\{v \in C^{1}[0,1]:\left.v\right|_{\left.\left.\right|_{\left.x_{k-1}, x_{k}\right]} \in P_{3}, k=1, \ldots, N_{x}\right\},}\right. \\
\mathscr{M}_{y} & =\left\{v \in C^{1}[0,1]:\left.v\right|_{\left[y_{l-1}, y_{l}\right]} \in P_{3}, l=1, \ldots, N_{y}\right\},
\end{aligned}
$$

where $P_{3}$ denotes the set of polynomials of degree $\leq 3$, and let

$$
\begin{aligned}
\mathscr{M}_{x}^{0}=\left\{v \in \mathscr{M}_{x}: v(0)\right. & =v(1)=0\}, & \mathscr{M}_{y}^{0} & =\left\{v \in \mathscr{M}_{y}: v(0)=v(1)=0\right\}, \\
\mathscr{M} & =\mathscr{M}_{x} \otimes \mathscr{M}_{y}, & \mathscr{M}^{0} & =\mathscr{M}_{x}^{0} \otimes \mathscr{M}_{y}^{0} .
\end{aligned}
$$

Let $\mathscr{G}_{x}=\left\{\xi_{k, i}^{x}\right\}_{k, i=1}^{N_{x}, 2}, \mathscr{G}_{y}=\left\{\xi_{l, j}^{y}\right\}_{l, j=1}^{N_{y}, 2}$ be the sets of Gauss points

$$
\xi_{k, i}^{x}=x_{k-1}+h_{k}^{x} \xi_{i}, \quad \xi_{l, j}^{y}=y_{l-1}+h_{l}^{y} \xi_{j},
$$

where $\xi_{1}=(3-\sqrt{3}) / 6, \xi_{2}=(3+\sqrt{3}) / 6$, and let

$$
\mathscr{G}=\left\{\left(\xi^{x}, \xi^{y}\right): \xi^{x} \in \mathscr{G}_{x}, \xi^{y} \in \mathscr{G}_{y}\right\} .
$$

For $u, v$ defined on $\mathscr{G}$, let $(u, v)_{\mathscr{G}}$ and $\|u\|_{\mathscr{G}}$ be given by

$$
(u, v)_{\mathscr{G}}=\frac{1}{4} \sum_{k=1}^{N_{x}} \sum_{l=1}^{N_{y}} h_{k}^{x} h_{l}^{y} \sum_{i=1}^{2} \sum_{j=1}^{2}(u v)\left(\xi_{k, i}^{x}, \xi_{l, j}^{y}\right),
$$

and

$$
\|u\|_{\mathscr{G}}=(u, u)_{\mathscr{G}}^{1 / 2} .
$$

The formula defining $(\cdot, \cdot)_{\mathscr{G}}$ is obtained by applying to $\iint_{\Omega}(u v)(x, y) d x d y$ the composite two-point Gauss quadrature rule with respect to $x$ and $y$. Since Lemma 2.3 of [19] implies that each $v \in \mathscr{M}^{0}$ is uniquely defined by its values on $\mathscr{G}, \mathscr{M}^{0}$ can be regarded as a Hilbert space with $(\cdot, \cdot)_{\mathscr{G}}$ as an inner product.

In the following, $C^{p, q, r}(\bar{Q})$ denotes the set of all functions $v(x, y, t)$ such that $\partial^{i+j+k} v / \partial x^{i} \partial y^{j} \partial t^{k}$ is continuous on $\bar{Q}$ for all $0 \leq i \leq p, 0 \leq j \leq q$, and $0 \leq k \leq r$. If $v \in C^{p, q, r}(\bar{Q})$, then $\|v\|_{C^{p, q, r}(\bar{Q})}$ is defined by

$$
\|v\|_{C^{p, q, r}(\bar{Q})}=\max _{\substack{0 \leq i \leq p \quad 0 \leq j \leq q \\ 0 \leq k \leq r}} \max _{(x, y, t) \in \bar{Q}}\left|\frac{\partial^{i+j+k} v}{\partial x^{i} \partial y^{j} \partial t^{k}}(x, y, t)\right| .
$$

Also, $C\left([0, T], H^{l}(\Omega)\right)$ denotes the set of all functions $v \in C(\bar{Q}) \equiv C^{0,0,0}(\bar{Q})$ such that $v(\cdot, t) \in H^{l}(\Omega)$ for $t \in[0, T]$, and

$$
\|v\|_{C\left([0, T], H^{\prime}(\Omega)\right)} \equiv \max _{0 \leq t \leq T}\|v(\cdot, t)\|_{H^{\prime}(\Omega)}<\infty .
$$


2.2. Stability result. In this subsection, following the approach of [26, 27], we state and prove stability results for two-level and three-level schemes in Hilbert spaces.

Let $H$ be a finite-dimensional Hilbert space over the field of real numbers. Let $(\cdot, \cdot)$ and $\|\cdot\|=\sqrt{(\cdot, \cdot)}$ denote the inner product and norm in $H$, respectively. A linear operator $A$ from $H$ into $H$ is said to be selfadjoint, written as $A=A^{*}$, if $(A v, w)=(v, A w)$ for all $v, w \in H$. If $A$ is a linear operator from $H$ into $H$, then $A \geq 0$ means that $(A v, v) \geq 0$ for all $v \in H$. If $A$ and $B$ are two linear operators from $H$ into $H$, then $A \geq B(A \leq B)$ means that $A-B \geq 0(B-A \geq 0)$. In the following, $E$ denotes the identity operator in $H$ and $\tau$ is a positive number.

First we present a stability result for the two-level scheme

$$
B \frac{v^{n+1}-v^{n}}{\tau}+A^{(n)} v^{n}=w^{n}, \quad n=0,1, \ldots, J-1,
$$

where $B, A^{(n)}$ are linear operators from $H$ into $H$ and $v^{n}, w^{n} \in H$.

Theorem 2.1. Let $A^{(n)}=A_{0}^{(n)}+A_{1}^{(n)}, A_{0}^{(n)}=\left[A_{0}^{(n)}\right]^{*}$, and let

$$
\begin{gathered}
A_{0}^{(n)} \geq \varepsilon_{0} A_{0}, \\
A_{0}^{(n)}-A_{0}^{(n-1)} \leq \varepsilon_{1} \tau A_{0}, \\
\left\|A_{1}^{(n)} v\right\|^{2} \leq \varepsilon_{2}\left(A_{0} v, v\right), \quad v \in H, \\
B \geq \varepsilon_{3} E+\frac{\tau}{2} A_{0}^{(n)},
\end{gathered}
$$

where $A_{0} \geq 0$ is a linear operator from $H$ into $H, \varepsilon_{0}, \varepsilon_{3}$ are positive constants, and $\varepsilon_{1}, \varepsilon_{2}$ are nonnegative constants. If $v^{n}, w^{n} \in H$ satisfy (2.1), then

$$
\max _{0 \leq n \leq J}\left(A_{0} v^{n}, v^{n}\right) \leq M\left[\left(A_{0}^{(0)} v^{0}, v^{0}\right)+\frac{\tau}{\varepsilon_{3}} \sum_{n=0}^{J-1}\left\|w^{n}\right\|^{2}\right],
$$

where $M=\varepsilon_{0}^{-1} e^{\left[\left(\varepsilon_{2}+\varepsilon_{1} \varepsilon_{3}\right) /\left(\varepsilon_{0} \varepsilon_{3}\right)\right] \tau J}$.

Proof. Since $v^{n}=(1 / 2)\left(v^{n+1}+v^{n}\right)-(\tau / 2) v_{t}^{n}$, where

$$
v_{t}^{n}=\frac{v^{n+1}-v^{n}}{\tau},
$$

equation (2.1) may be rewritten as

$$
\begin{aligned}
{\left[B-\frac{\tau}{2} A_{0}^{(n)}\right] v_{t}^{n}+\frac{1}{2} A_{0}^{(n)}\left(v^{n+1}+v^{n}\right)=w^{n} } & -A_{1}^{(n)} v^{n}, \\
n & =0,1, \ldots, J-1 .
\end{aligned}
$$

It follows from the Cauchy-Schwarz inequality, the inequality $\alpha \beta \leq \alpha^{2} /\left(2 \varepsilon_{3}\right)+$ $\varepsilon_{3} \beta^{2} / 2$, and (2.4) that

$$
\left(w^{n}, v_{t}^{n}\right)-\left(A_{1}^{(n)} v^{n}, v_{t}^{n}\right) \leq \varepsilon_{3}\left\|v_{t}^{n}\right\|^{2}+\frac{1}{2 \varepsilon_{3}}\left\|w^{n}\right\|^{2}+\frac{\varepsilon_{2}}{2 \varepsilon_{3}}\left(A_{0} v^{n}, v^{n}\right)
$$


Taking the inner product of each side of (2.8) with $2 \tau v_{t}^{n}$ and using (2.9) and (2.5), we obtain

$$
\tau\left(A_{0}^{(n)}\left(v^{n+1}+v^{n}\right), v_{t}^{n}\right) \leq \frac{\tau}{\varepsilon_{3}}\left\|w^{n}\right\|^{2}+\frac{\varepsilon_{2}}{\varepsilon_{3}} \tau\left(A_{0} v^{n}, v^{n}\right) .
$$

Since $A_{0}^{(n)}=\left[A_{0}^{(n)}\right]^{*}$, it is easy to verify that

$$
\begin{aligned}
& \tau \sum_{n=0}^{k-1}\left(A_{0}^{(n)}\left(v^{n+1}+v^{n}\right), v_{t}^{n}\right) \\
& \quad=\left(A_{0}^{(k-1)} v^{k}, v^{k}\right)-\sum_{n=1}^{k-1}\left(\left[A_{0}^{(n)}-A_{0}^{(n-1)}\right] v^{n}, v^{n}\right)-\left(A_{0}^{(0)} v^{0}, v^{0}\right) .
\end{aligned}
$$

Summing both sides of $(2.10)$ from $n=0$ to $k-1$, where $k=1, \ldots, J$, and using (2.11), (2.3), $A_{0} \geq 0$, and (2.2), we obtain

$$
\left(A_{0} v^{k}, v^{k}\right) \leq \varepsilon_{0}^{-1}\left(A_{0}^{(0)} v^{0}, v^{0}\right)+\frac{\tau}{\varepsilon_{0} \varepsilon_{3}} \sum_{n=0}^{k-1}\left\|w^{n}\right\|^{2}+\frac{\varepsilon_{2}+\varepsilon_{1} \varepsilon_{3}}{\varepsilon_{0} \varepsilon_{3}} \tau \sum_{n=0}^{k-1}\left(A_{0} v^{n}, v^{n}\right)
$$

for $k=0, \ldots, J$. The bound (2.6) now follows from the discrete Gronwall inequality [24], which states that if $\alpha_{k}, \beta_{k}, k=0, \ldots, J$, are nonnegative numbers, $\beta_{k} \leq \beta_{k+1}$, and

$$
\alpha_{k} \leq \beta_{k}+\gamma \tau \sum_{n=0}^{k-1} \alpha_{n}, \quad k=0, \ldots, J,
$$

where $\gamma$ is a positive constant, then

$$
\alpha_{n} \leq e^{\gamma \tau n} \beta_{n}, \quad n=0, \ldots, J .
$$

Next, we present a stability result for the three-level scheme

$$
\begin{aligned}
B \frac{v^{n+1}-v^{n-1}}{2 \tau}+\tau^{2} R \frac{v^{n+1}-2 v^{n}+v^{n-1}}{\tau^{2}}+A^{(n)} v^{n} & =w^{n}, \\
n & =1, \ldots, J-1,
\end{aligned}
$$

where $B, R, A^{(n)}$ are linear operators from $H$ into $H$ and $v^{n}, w^{n} \in H$.

Theorem 2.2. Let $A^{(n)}=A_{0}^{(n)}+A_{1}^{(n)}, A_{0}^{(n)}=\left[A_{0}^{(n)}\right]^{*}, R=R^{*}$, and let

$$
A_{0}^{(n)} \geq \varepsilon_{0} A_{0},
$$

$$
\begin{gathered}
\varepsilon_{1} \tau\left[A_{0}^{(n-1)}-4 R\right] \leq A_{0}^{(n)}-A_{0}^{(n-1)} \leq \varepsilon_{1} \tau A_{0}^{(n-1)}, \\
\left\|A_{1}^{(n)} v\right\|^{2} \leq \varepsilon_{2}\left(A_{0} v, v\right), \quad v \in H, \\
B \geq \varepsilon_{3} E \\
R \geq \frac{1+\varepsilon_{4}}{4} A_{0}^{(n)}
\end{gathered}
$$


where $A_{0} \geq 0$ is a linear operator from $H$ into $H, \varepsilon_{0}, \varepsilon_{3}, \varepsilon_{4}$ are positive constants and $\varepsilon_{1}, \varepsilon_{2}$ are nonnegative constants. If $v^{n}, w^{n} \in H$ satisfy (2.13), then

$$
\begin{aligned}
\max _{0 \leq n \leq J}\left(A_{0} v^{n}, v^{n}\right) \leq M\left[\left(A_{0}^{(1)} v^{0}\right.\right. & \left., v^{0}\right)+\left(R v_{0}, v_{0}\right)+\left(R v_{1}, v_{1}\right) \\
& \left.+\frac{\varepsilon_{2} \tau}{\varepsilon_{3}}\left(A_{0} v^{1}, v^{1}\right)+\frac{\tau}{\varepsilon_{3}} \sum_{n=1}^{J-1}\left\|w^{n}\right\|^{2}\right],
\end{aligned}
$$

where $M=4\left[\left(1+\varepsilon_{4}\right) /\left(\varepsilon_{0} \varepsilon_{4}\right)\right] e^{\left[\varepsilon_{1}+\left\{\varepsilon_{2}\left(1+\varepsilon_{4}\right)\right\} /\left(\varepsilon_{0} \varepsilon_{3} \varepsilon_{4}\right)\right] \tau J}$.

Proof. Since $v^{n}=(1 / 2)\left(v^{n+1}+v^{n-1}\right)-(\tau / 2)\left(v_{t}^{n}-v_{t}^{n-1}\right)$, where $v_{t}^{n}$ is defined by (2.7), equation (2.13) may be written as

$$
\begin{aligned}
B v_{i}^{n}+\tau\left[R-\frac{1}{2} A_{0}^{(n)}\right]\left(v_{t}^{n}-v_{t}^{n-1}\right)+\frac{1}{2} A_{0}^{(n)}\left(v^{n+1}+v^{n-1}\right) & =w^{n}-A_{1}^{(n)} v^{n}, \\
n & =1, \ldots, J-1,
\end{aligned}
$$

where

$$
v_{\tilde{t}}^{n}=\frac{v^{n+1}-v^{n-1}}{2 \tau}
$$

Taking the inner product of each side with $2 \tau v_{\tilde{t}}^{n}=\tau\left(v_{t}^{n}+v_{t}^{n-1}\right)$, we obtain

$$
\begin{aligned}
& 2 \tau\left(B v_{\tilde{t}}^{n}, v_{\tilde{t}}^{n}\right)+\tau^{2}\left(\left[R-\frac{1}{2} A_{0}^{(n)}\right]\left(v_{t}^{n}-v_{t}^{n-1}\right), v_{t}^{n}+v_{t}^{n-1}\right) \\
& \quad+\frac{1}{2}\left(A_{0}^{(n)}\left(v^{n+1}+v^{n-1}\right), v^{n+1}-v^{n-1}\right)=2 \tau\left(w^{n}-A_{1}^{(n)} v^{n}, v_{\tilde{t}}^{n}\right)
\end{aligned}
$$

Since $R=R^{*}$ and $A_{0}^{(n)}=\left[A_{0}^{(n)}\right]^{*}$, it is easy to verify that

$$
\begin{aligned}
& \left(\left[R-\frac{1}{2} A_{0}^{(n)}\right]\left(v_{t}^{n}-v_{t}^{n-1}\right), v_{t}^{n}+v_{t}^{n-1}\right) \\
& \quad=\left(\left[R-\frac{1}{2} A_{0}^{(n)}\right] v_{t}^{n}, v_{t}^{n}\right)-\left(\left[R-\frac{1}{2} A_{0}^{(n)}\right] v_{t}^{n-1}, v_{t}^{n-1}\right)
\end{aligned}
$$

and

$$
\begin{aligned}
\left(A_{0}^{(n)}\left(v^{n+1}+v^{n-1}\right), v^{n+1}-v^{n-1}\right) \\
=\frac{1}{2}\left(A_{0}^{(n)}\left(v^{n+1}+v^{n}\right), v^{n+1}+v^{n}\right)+\frac{\tau^{2}}{2}\left(A_{0}^{(n)} v_{t}^{n}, v_{t}^{n}\right) \\
\quad-\frac{1}{2}\left(A_{0}^{(n)}\left(v^{n}+v^{n-1}\right), v^{n}+v^{n-1}\right)-\frac{\tau^{2}}{2}\left(A_{0}^{(n)} v_{t}^{n-1}, v_{t}^{n-1}\right) .
\end{aligned}
$$

From (2.20)-(2.22) it follows that, for $n=1, \ldots, J-1$,

$$
2 \tau\left(B v_{\tilde{t}}^{n}, v_{\tilde{t}}^{n}\right)+\left\|\left|v^{n}\right|\right\|_{n}^{2}=\left\|\left|v^{n-1}\right|\right\|_{n}^{2}+2 \tau\left(w^{n}-A_{1}^{(n)} v^{n}, v_{\tilde{t}}^{n}\right),
$$

where for $k=n, n-1$,

$$
\left\|v^{k} \mid\right\|_{n}^{2}=\frac{1}{4}\left(A_{0}^{(n)}\left(v^{k+1}+v^{k}\right), v^{k+1}+v^{k}\right)+\tau^{2}\left(\left[R-\frac{1}{4} A_{0}^{(n)}\right] v_{t}^{k}, v_{t}^{k}\right) .
$$


By means of (2.15) it is easy to see that, for $n=2, \ldots, J-1$,

$$
\begin{aligned}
\left\|v^{n-1}\right\| \|_{n}^{2}= & \left\|v^{n-1} \mid\right\|_{n-1}^{2}+\frac{1}{4}\left(\left[A_{0}^{(n)}-A_{0}^{(n-1)}\right]\left(v^{n}+v^{n-1}\right), v^{n}+v^{n-1}\right) \\
& +\frac{\tau^{2}}{4}\left(\left[A_{0}^{(n-1)}-A_{0}^{(n)}\right] v_{t}^{n-1}, v_{t}^{n-1}\right) \leq\left(1+\varepsilon_{1} \tau\right)\left\|v^{n-1}\right\|_{n-1}^{2} .
\end{aligned}
$$

Also, as in (2.9), we obtain

$$
\left(w^{n}, v_{i}^{n}\right)-\left(A_{1}^{(n)} v^{n}, v_{t}^{n}\right) \leq \varepsilon_{3}\left\|v_{i}^{n}\right\|^{2}+\frac{1}{2 \varepsilon_{3}}\left\|w^{n}\right\|^{2}+\frac{\varepsilon_{2}}{2 \varepsilon_{3}}\left(A_{0} v^{n}, v^{n}\right)
$$

Therefore, using (2.24), (2.25) and (2.17) in (2.23), and then summing both sides of the resulting inequality from $n=1$ to $k$, where $k=1, \ldots, J-1$, we obtain

$$
\begin{aligned}
\left\|v^{k}\right\| \|_{k}^{2} \leq & \left\|v^{0}\right\|\left\|_{1}^{2}+\varepsilon_{1} \tau \sum_{n=1}^{k-1}\right\| v^{n}\|\|_{n}^{2}+\frac{\tau}{\varepsilon_{3}} \sum_{n=1}^{k}\left\|w^{n}\right\|^{2} \\
& +\frac{\varepsilon_{2} \tau}{\varepsilon_{3}} \sum_{n=1}^{k}\left(A_{0} v^{n}, v^{n}\right) .
\end{aligned}
$$

Since $A_{0}^{(n)}=\left[A_{0}^{(n)}\right]^{*}$, it is easy to verify that

$$
\left\|v^{n}\right\| \|_{n}^{2}=\left(A_{0}^{(n)} v^{n+1}, v^{n+1}\right)-\tau\left(A_{0}^{(n)} v^{n+1}, v_{t}^{n}\right)+\tau^{2}\left(R v_{t}^{n}, v_{t}^{n}\right) .
$$

Also, since $A_{0}^{(n)} \geq 0$, it follows from the Cauchy-Schwarz inequality, the inequality $\alpha \beta \leq \tilde{\varepsilon} \alpha^{2}+\beta^{2} /(4 \tilde{\varepsilon})$ and (2.18), that

$$
\begin{aligned}
\tau\left(A_{0}^{(n)} v^{n+1}, v_{t}^{n}\right) & \leq \tilde{\varepsilon}\left(A_{0}^{(n)} v^{n+1}, v^{n+1}\right)+\frac{\tau^{2}}{4 \tilde{\varepsilon}}\left(A_{0}^{(n)} v_{t}^{n}, v_{t}^{n}\right) \\
& \leq \tilde{\varepsilon}\left(A_{0}^{(n)} v^{n+1}, v^{n+1}\right)+\frac{\tau^{2}}{\tilde{\varepsilon}\left(1+\varepsilon_{4}\right)}\left(R v_{t}^{n}, v_{t}^{n}\right) .
\end{aligned}
$$

From (2.27) and (2.28), it follows that

(2.29) $\left\|v^{n}\right\| \|_{n}^{2} \geq(1-\tilde{\varepsilon})\left(A_{0}^{(n)} v^{n+1}, v^{n+1}\right)+\tau^{2}\left[1-\frac{1}{\tilde{\varepsilon}\left(1+\varepsilon_{4}\right)}\right]\left(R v_{t}^{n}, v_{t}^{n}\right)$.

Choosing $\tilde{\varepsilon}=\left(1+\varepsilon_{4}\right)^{-1}$ in (2.29) and using (2.14), we obtain

$$
\left(A_{0} v^{n+1}, v^{n+1}\right) \leq \frac{1+\varepsilon_{4}}{\varepsilon_{0} \varepsilon_{4}}\left\|v^{n}\right\|_{n}^{2}, \quad n=1, \ldots, J-1 .
$$

By arguments similar to those used in (2.27) and (2.28), it follows that

$$
\left.\left\|v^{0}\right\|\right|_{1} ^{2}=\left(A_{0}^{(1)} v^{0}, v^{0}\right)+\tau\left(A_{0}^{(1)} v_{t}^{0}, v^{0}\right)+\tau^{2}\left(R v_{t}^{0}, v_{t}^{0}\right),
$$

and

$$
\tau\left(A_{0}^{(1)} v_{t}^{0}, v^{0}\right) \leq\left(A_{0}^{(1)} v^{0}, v^{0}\right)+\tau^{2}\left(R v_{t}^{0}, v_{t}^{0}\right),
$$

since $A_{0}^{(1)} \leq 4 R$. Therefore,

$$
\left\|v^{0}\right\| \|_{1}^{2} \leq 2\left[\left(A_{0}^{(1)} v^{0}, v^{0}\right)+\tau^{2}\left(R v_{t}^{0}, v_{t}^{0}\right)\right] .
$$


Using (2.26), (2.30) and (2.31), we obtain

$$
\begin{aligned}
\left\|v^{k}\right\| \|_{k}^{2} \leq & 2\left[\left(A_{0}^{(1)} v^{0}, v^{0}\right)+\tau^{2}\left(R v_{t}^{0}, v_{t}^{0}\right)\right]+\frac{\varepsilon_{2} \tau}{\varepsilon_{3}}\left(A_{0} v^{1}, v^{1}\right)+\frac{\tau}{\varepsilon_{3}} \sum_{n=1}^{k}\left\|w^{n}\right\|^{2} \\
& +\left[\varepsilon_{1}+\frac{\varepsilon_{2}\left(1+\varepsilon_{4}\right)}{\varepsilon_{0} \varepsilon_{3} \varepsilon_{4}}\right] \tau \sum_{n=1}^{k-1}\left\|v^{n}\right\|_{n}^{2} .
\end{aligned}
$$

Since $\tau^{2}\left(R v_{t}^{0}, v_{t}^{0}\right) \leq 2\left[\left(R v^{0}, v^{0}\right)+\left(R v^{1}, v^{1}\right)\right]$, Gronwall's inequality (2.12) and (2.30) imply that for $n=2, \ldots, J,\left(A_{0} v^{n}, v^{n}\right)$ is bounded by the righthand side of (2.19). Inequalities (2.14) and (2.18) show that $\left(A_{0} v^{n}, v^{n}\right) \leq$ $M\left(R v^{n}, v^{n}\right)$ for $n=0,1$, and hence (2.19) follows.

\section{TWO-LEVEL SCHEMES}

In this section, we present and analyze three two-level piecewise Hermite bicubic orthogonal spline collocation schemes for the approximate solution of the parabolic problem (1.1). We divide the interval $[0, T]$ using the partition $\left\{t_{n}\right\}_{n=0}^{J}$, where $t_{n}=n \tau$ and $\tau=T / J$. Throughout this paper, $L^{n}$ is the elliptic differential operator defined by the right-hand side of (1.2) with $t=t_{n}$. Also, $C$ denotes a generic positive constant that is independent of $h$ and $\tau$.

3.1. Laplace-modified Euler method. Assume that with respect to the spatial variables, (1.1) is discretized by orthogonal spline collocation with piecewise Hermite bicubics. If a forward finite difference quotient is used for the time discretization, then the resulting discrete collocation scheme is only conditionally stable. Perturbing this scheme, we obtain the LM Euler method, in which the approximate solution $u_{h}^{n} \in \mathscr{M}, n=1, \ldots, J$, is required to satisfy

$$
\begin{aligned}
{\left[(1-\tau \lambda \Delta) \frac{u_{h}^{n+1}-u_{h}^{n}}{\tau}+L^{n} u_{h}^{n}\right](\xi)=} & f\left(\xi, t_{n}\right), \\
& \xi \in \mathscr{G}, n=0,1, \ldots, J-1,
\end{aligned}
$$

where $u_{h}^{0} \in \mathscr{M}$ and $\left.u_{h}^{n}\right|_{\partial \Omega}, n=1, \ldots, J$, are assumed to be given. The functions $u_{h}^{0}$ and $\left.u_{h}^{n}\right|_{\partial \Omega}$ can be prescribed by approximating the initial and boundary conditions of (1.1) by either Hermite or Gauss piecewise bicubic and cubic interpolations, respectively. For example, with $g_{2}^{n}(\cdot)=g_{2}\left(\cdot, t_{n}\right)$, in Hermite interpolation we require that

$$
\begin{aligned}
& \frac{\partial^{i+j}\left(u_{h}^{0}-g_{1}\right)}{\partial x^{i} \partial y^{j}}\left(x_{k}, y_{l}\right)=0, \quad i, j=0,1,0 \leq k \leq N_{x}, 0 \leq l \leq N_{y} \\
& \frac{\partial^{i}\left(u_{h}^{n}-g_{2}^{n}\right)}{\partial x^{i}}\left(x_{k}, \alpha\right)=0, \quad i=0,1,0 \leq k \leq N_{x}, \alpha=0,1, n \geq 1, \\
& \frac{\partial^{i}\left(u_{h}^{n}-g_{2}^{n}\right)}{\partial y^{i}}\left(\alpha, y_{l}\right)=0, \quad i=0,1,0 \leq l \leq N_{y}, \alpha=0,1, n \geq 1
\end{aligned}
$$


whereas using Gauss interpolation, we have

$$
\begin{gathered}
\left(u_{h}^{0}-g_{1}\right)(\xi)=0, \quad \xi \in \mathscr{G}, \\
\left(u_{h}^{0}-g_{1}\right)\left(\xi^{x}, \alpha\right)=0, \quad \alpha=0,1, \quad \xi^{x} \in \mathscr{G}_{x}, \\
\left(u_{h}^{0}-g_{1}\right)\left(\alpha, \xi^{y}\right)=0, \quad \alpha=0,1, \quad \xi^{y} \in \mathscr{G}_{y}, \\
\left(u_{h}^{0}-g_{1}\right)(\alpha, \beta)=0, \quad \alpha, \beta=0,1, \\
\left(u_{h}^{n}-g_{2}^{n}\right)\left(\xi^{x}, \alpha\right)=0, \quad \alpha=0,1, \quad \xi^{x} \in \mathscr{G}_{x}, \quad n \geq 1, \\
\left(u_{h}^{n}-g_{2}^{n}\right)\left(\alpha, \xi^{y}\right)=0, \quad \alpha=0,1, \quad \xi^{y} \in \mathscr{G}_{y}, \quad n \geq 1, \\
\left(u_{h}^{n}-g_{2}^{n}\right)(\alpha, \beta)=0, \quad \alpha, \beta=0,1, \quad n \geq 1 .
\end{gathered}
$$

For computational purposes, it is more convenient to use Gauss interpolation, since it does not require the knowledge or evaluation of first partial derivatives of $g_{1}$ and $g_{2}$. However, in this paper we consider Hermite interpolation, since the convergence analysis is much simpler for this type of boundary condition approximation (cf. [2]).

Let $u$ be a sufficiently smooth function defined on $\bar{Q}$. For each $t \in[0, T]$, the comparison function $u_{\mathscr{L}}(\cdot, t) \in \mathscr{M}$ is defined as the piecewise Hermite bicubic interpolant of $u(\cdot, t)$, that is,

$$
\frac{\partial^{i+j}\left(u_{\mathscr{H}}-u\right)}{\partial x^{i} \partial y^{j}}\left(x_{k}, y_{l}, t\right)=0, \quad i, j=0,1,0 \leq k \leq N_{x}, 0 \leq l \leq N_{y} .
$$

In the following, we write $u^{n}$ and $u_{\mathscr{H}}^{n}$ in place of $u\left(\cdot, t_{n}\right)$ and $u_{\mathscr{L}}\left(\cdot, t_{n}\right)$, respectively. For $n=0, \ldots, J-1$ and $\xi \in \mathscr{G}$, the truncation error $T_{u}^{n}(\xi)$ of the scheme (3.1) is defined by

$$
T_{u}^{n}(\xi)=\left[\left(\frac{\partial u}{\partial t}\right)^{n}-(1-\tau \lambda \Delta) \frac{u_{\mathscr{H}}^{n+1}-u_{\mathscr{H}}^{n}}{\tau}+L^{n}\left(u^{n}-u_{\mathscr{H}}^{n}\right)\right](\xi) .
$$

If $u$ is a solution of $(1.1)$, then $\left[(\partial u / \partial t)^{n}+L^{n} u^{n}\right](\xi)=f\left(\xi, t_{n}\right)$, and hence $T_{u}^{n}(\xi)$ indicates by how much $u_{\mathscr{H}}$ fails to satisfy (3.1). The following lemma gives a bound on a discrete norm of the truncation error $T_{u}^{n}$.

Lemma 3.1. Assume $a_{i}, b_{i}, i=1,2$, and $c$ are such that $a_{1} \in C^{1,0,0}(\bar{Q})$, $a_{2} \in C^{0,1,0}(\bar{Q})$, and $b_{1}, b_{2}, c \in C(\bar{Q})$. If $u \in C\left([0, T], H^{5}(\Omega)\right) \cap C^{2,0,1}(\bar{Q}) \cap$ $C^{0,2,1}(\bar{Q}) \cap C^{0,0,2}(\bar{Q})$ and $\partial u / \partial t \in C\left([0, T], H^{3}(\Omega)\right)$, then

$$
\begin{aligned}
\tau \sum_{n=0}^{J-1}\left\|T_{u}^{n}\right\|_{\mathscr{G}}^{2} \leq C\{ & \tau^{2}\left[\|u\|_{C^{0,0,2}(\bar{Q})}^{2}+\lambda^{2}\left(\|u\|_{C^{2,0,1}(\bar{Q})}^{2}+\|u\|_{C^{0,2,1}(\bar{Q})}^{2}\right)\right] \\
& \left.+h^{6}\left[\left\|\frac{\partial u}{\partial t}\right\|_{C\left([0, T], H^{3}(\Omega)\right)}^{2}+\left(1+\lambda^{2}\right)\|u\|_{C\left([0, T], H^{5}(\Omega)\right)}^{2}\right]\right\} .
\end{aligned}
$$

Proof. It follows from Lemma 4.2 of [2] and its proof that, for $k=0,1,2$,

$$
\left\|\frac{\partial^{k}\left(u-u_{\mathscr{H}}\right)}{\partial x^{k-i} \partial y^{i}}\right\|_{\mathscr{G}} \leq C h^{3}\|u\|_{H^{3+k}}, \quad i=0, k
$$

Therefore,

$$
\left\|L^{n}\left(u^{n}-u_{\mathscr{H}}^{n}\right)\right\|_{\mathscr{G}}^{2} \leq C h^{6}\|u\|_{C\left([0, T], H^{5}(\Omega)\right)}^{2}
$$


Taylor's theorem gives

$$
\Delta\left(u^{n+1}-u^{n}\right)(\xi)=\tau \frac{\partial(\Delta u)}{\partial t}\left(\xi, \bar{t}_{\xi, n}\right), \quad \xi \in \mathscr{G}, \quad t_{n} \leq \bar{t}_{\xi, n} \leq t_{n+1},
$$

and hence the triangle inequality and (3.9) imply

$$
\begin{aligned}
& \left\|\Delta\left(u_{\mathscr{X}}^{n+1}-u_{\mathscr{X}}^{n}\right)\right\|_{\mathscr{G}}^{2} \\
& \quad \leq C\left(h^{6}\|u\|_{C\left([0, T], H^{5}(\Omega)\right)}^{2}+\tau^{2}\left[\|u\|_{C^{2,0,1}(\bar{Q})}^{2}+\|u\|_{C^{0,2,1}(\bar{Q})}^{2}\right]\right) .
\end{aligned}
$$

By Taylor's theorem,

$$
\left(\frac{\partial u}{\partial t}\right)^{n}(\xi)-\frac{u^{n+1}-u^{n}}{\tau}(\xi)=-\frac{\tau}{2} \frac{\partial^{2} u}{\partial t^{2}}\left(\xi, \tilde{t}_{\xi, n}\right), \quad \xi \in \mathscr{G}, \quad t_{n} \leq \tilde{t}_{\xi, n} \leq t_{n+1},
$$

and hence

$$
\left\|\left(\frac{\partial u}{\partial t}\right)^{n}-\frac{u^{n+1}-u^{n}}{\tau}\right\|_{\mathscr{G}}^{2} \leq C \tau^{2}\|u\|_{C^{0,0,2}(\bar{Q})}^{2} .
$$

Also, since $\partial u_{\mathscr{L}} / \partial t=(\partial u / \partial t)_{\mathscr{H}}$,

$$
\begin{aligned}
& \left\|\frac{u^{n+1}-u^{n}}{\tau}-\frac{u_{\mathscr{C}}^{n+1}-u_{\mathscr{C}}^{n}}{\tau}\right\|_{\mathscr{G}}^{2}=\left\|\frac{1}{\tau} \int_{n \tau}^{(n+1) \tau} \frac{\partial}{\partial t}\left(u-u_{\mathscr{C}}\right)(\cdot, s) d s\right\|_{\mathscr{G}}^{2} \\
& \quad \leq \frac{1}{\tau} \int_{n \tau}^{(n+1) \tau}\left\|\left[\frac{\partial u}{\partial t}-\left(\frac{\partial u}{\partial t}\right)_{\mathscr{H}}\right](\cdot, s)\right\|_{\mathscr{G}}^{2} d s \\
& \leq C h^{6}\left\|\frac{\partial u}{\partial t}\right\|_{C\left([0, T], H^{3}(\Omega)\right)}^{2},
\end{aligned}
$$

where the last inequality follows from (3.9). Finally, (3.8) is obtained by combining (3.10)-(3.13).

We show that if the constant $\lambda$ is sufficiently large, then the scheme (3.1) is unconditionally stable with respect to the initial condition and the right-hand side.

Lemma 3.2. Assume $a_{1} \in C^{5,0,0}(\bar{Q}), a_{2} \in C^{0,5,0}(\bar{Q}), b_{1}, b_{2}, c \in C(\bar{Q})$, and

$$
0<a_{\min } \leq a_{1}(x, y, t), a_{2}(x, y, t) \leq a_{\max }, \quad(x, y, t) \in \bar{Q} .
$$

Also assume that $a_{i}, i=1,2$, satisfy a Lipschitz condition with respect to $t$, that is, there is a constant $K>0$ such that for $i=1,2$,

$$
\begin{array}{r}
\left|a_{i}\left(x, y, t_{1}\right)-a_{i}\left(x, y, t_{2}\right)\right| \leq K\left|t_{1}-t_{2}\right|, \\
\quad(x, y) \in \bar{\Omega}, t_{1}, t_{2} \in[0, T] .
\end{array}
$$

Let $v^{n}, w^{n} \in \mathscr{M}^{0}$ be such that

$$
\begin{aligned}
{\left[(1-\tau \lambda \Delta) \frac{v^{n+1}-v^{n}}{\tau}+L^{n} v^{n}\right](\xi) } & =w^{n}(\xi), \\
\xi & \in \mathscr{G}, \quad n=0, \ldots, J-1,
\end{aligned}
$$


where

$$
\lambda \geq a_{\max } / 2
$$

Then

$$
\max _{0 \leq n \leq J}\left\|v^{n}\right\|_{H^{1}(\Omega)}^{2} \leq M\left[a_{\max }\left(-\Delta v^{0}, v^{0}\right)_{\mathscr{G}}+\tau \sum_{n=0}^{J-1}\left\|w^{n}\right\|_{\mathscr{G}}^{2}\right],
$$

where $M=C a_{\min }^{-1} e^{\left[C\left(\alpha+\beta_{1}+\beta_{2}+\gamma\right)^{2}+K\right] T / a_{\min }}, C$ is a positive constant independent of $a_{i}, b_{i}, i=1,2, c, h, \tau$, and $\alpha, \beta_{i}, i=1,2, \gamma$ are such that

$$
\begin{aligned}
& \left\|\frac{\partial^{l} a_{1}}{\partial x^{l}}\right\|_{C(\bar{Q})},\left\|\frac{\partial^{l} a_{2}}{\partial y^{l}}\right\|_{C(\bar{Q})} \leq \alpha, \quad 0 \leq l \leq 5, \\
& \left\|b_{i}\right\|_{C(\bar{Q})} \leq \beta_{i}, \quad i=1,2, \quad\|c\|_{C(\bar{Q})} \leq \gamma .
\end{aligned}
$$

Proof. Let $L_{h}^{n}, n=0, \ldots, J-1$, and $\Delta_{h}$ be the operators from $\mathscr{M}^{0}$ into $\mathscr{M}^{0}$ defined by

$$
\left(L_{h}^{n} v\right)(\xi)=L^{n} v(\xi), \quad\left(\Delta_{h} v\right)(\xi)=\Delta v(\xi), \quad \xi \in \mathscr{G} .
$$

It is well known (see, for example, (2.5) of [2]), that $-\Delta_{h}$ is a positive definite operator from $\mathscr{M}^{0}$ into $\mathscr{M}^{0}$, that is

$$
C\|v\|_{\mathscr{G}}^{2} \leq\left(-\Delta_{h} v, v\right)_{\mathscr{G}}, \quad v \in \mathscr{M}^{0} .
$$

The operator form of $(3.15)$ is given by $(2.1)$, where

$$
A^{(n)}=L_{h}^{n}, \quad B=E-\tau \lambda \Delta_{h} .
$$

Employing the approach of the proof of Theorem 4.2 in [2] and using (2.6) of [25], Lemma 3.2 and (3.2) of [19], and (3.19), we can show that

$$
\left(A^{(n)} v, w\right)_{\mathscr{G}}=\mathscr{A}_{0}^{(n)}(v, w)+\mathscr{A}_{1}^{(n)}(v, w),
$$

where $\mathscr{A}_{i}^{(n)}, i=0,1$, are real-valued bilinear forms on $\mathscr{M}^{0} \times \mathscr{M}^{0}$ such that

$$
\begin{gathered}
\mathscr{A}_{0}^{(n)}(v, w)=\mathscr{A}_{0}^{(n)}(w, v), \\
a_{\min }\left(-\Delta_{h} v, v\right)_{\mathscr{G}} \leq \mathscr{A}_{0}^{(n)}(v, v) \leq a_{\max }\left(-\Delta_{h} v, v\right)_{\mathscr{G}}, \\
\left|\mathscr{A}_{0}^{(n)}(v, v)-\mathscr{A}_{0}^{(n-1)}(v, v)\right| \leq K \tau\left(-\Delta_{h} v, v\right)_{\mathscr{G}}, \\
\left|\mathscr{A}_{1}^{(n)}(v, w)\right| \leq C \delta\left(-\Delta_{h} v, v\right)_{\mathscr{G}}^{1 / 2}\|w\|_{\mathscr{G}},
\end{gathered}
$$

where $\delta$ in (3.24) is given by

$$
\delta=\alpha+\beta_{1}+\beta_{2}+\gamma .
$$

Let $A_{0}^{(n)}$ be the operator from $\mathscr{M}^{0}$ into $\mathscr{M}^{0}$ defined as follows: for $v \in \mathscr{M}^{0}$, let $A_{0}^{(n)} v$ be the element in $\mathscr{M}^{0}$ such that

$$
\left(A_{0}^{(n)} v, w\right)_{\mathscr{G}}=\mathscr{A}_{0}^{(n)}(v, w), \quad w \in \mathscr{M}^{0} .
$$


The Riesz theorem guarantees that $A_{0}^{(n)}$ is well defined, since $\mathscr{M}^{0}$ is a finitedimensional Hilbert space and hence for fixed $v, \mathscr{A}_{0}^{(n)}(v, \cdot)$ is a bounded linear functional on $\mathscr{M}^{0}$. Let $A_{1}^{(n)}$ be the operator from $\mathscr{M}^{0}$ into $\mathscr{M}^{0}$ such that

$$
A^{(n)}=A_{0}^{(n)}+A_{1}^{(n)} .
$$

It follows from (3.21), (3.22), and (3.26) that

$$
a_{\min }\left(-\Delta_{h}\right) \leq A_{0}^{(n)}=\left[A_{0}^{(n)}\right]^{*} \leq a_{\max }\left(-\Delta_{h}\right),
$$

where the inequalities are to be understood with respect to the inner product $(\cdot, \cdot)_{\mathscr{G}}$ in $\mathscr{M}^{0}$. Moreover, by (3.23),

$$
K \tau \Delta_{h} \leq A_{0}^{(n)}-A_{0}^{(n-1)} \leq K \tau\left(-\Delta_{h}\right)
$$

Further, (3.27), (3.20), (3.26), and then (3.24) imply that

$$
\left\|A_{1}^{(n)} v\right\|_{\mathscr{G}}^{2}=\mathscr{A}_{1}\left(v, A_{1}^{(n)} v\right) \leq C \delta\left(-\Delta_{h} v, v\right)_{\mathscr{G}}^{1 / 2}\left\|A_{1}^{(n)} v\right\|_{\mathscr{G}}
$$

from which it follows that

$$
\left\|A_{1}^{(n)} v\right\|_{\mathscr{G}}^{2} \leq C \delta^{2}\left(-\Delta_{h} v, v\right)_{\mathscr{G}}, \quad v \in \mathscr{M}^{0} .
$$

Using (3.16) and (3.28), we also easily verify that

$$
B \geq E+\frac{\tau}{2} A_{0}^{(n)} .
$$

Therefore, (3.27)-(3.31) imply that all assumptions of Theorem 2.1 are satisfied for $H=\mathscr{M}^{0}$ with $A_{0}=-\Delta_{h}, \varepsilon_{0}=a_{\min }, \varepsilon_{1}=K, \varepsilon_{2}=C \delta^{2}, \varepsilon_{3}=1$. Hence, (3.17) follows from (2.6), (3.25), (3.28), (3.18), and the inequality (see (2.7) and (2.8) in [25])

$$
C\|v\|_{H^{1}(\Omega)}^{2} \leq\left(-\Delta_{h} v, v\right)_{\mathscr{G}} .
$$

Using the bound on the truncation error and the stability result, we prove the following convergence theorem.

Theorem 3.1. Assume that $a_{i}, b_{i}, i=1,2$, and $c$ satisfy the assumptions of Lemma 3.2 and that the solution $u$ of (1.1) satisfies the assumptions of Lemma 3.1. Let $\lambda$ satisfy (3.16), and let $u_{h}^{n} \in \mathscr{M}, n=1, \ldots, J$, be solutions of (3.1), where $u_{h}^{0} \in \mathscr{M}$ and $\left.u_{h}^{n}\right|_{\partial \Omega}, n=1, \ldots, J$, are given by (3.2), (3.3), respectively. Then

$$
\begin{aligned}
\max _{0 \leq n \leq J}\left\|u^{n}-u_{h}^{n}\right\|_{H^{1}(\Omega)} \leq C\left\{\tau\left[\|u\|_{C^{0,0,2}(\bar{Q})}+\|u\|_{C^{2,0,1}(\bar{Q})}+\|u\|_{C^{0,2,1}(\bar{Q})}\right]\right. \\
\left.+h^{3}\left[\left\|\frac{\partial u}{\partial t}\right\|_{C\left([0, T], H^{3}(\Omega)\right)}+\|u\|_{C\left([0, T], H^{5}(\Omega)\right)}\right]\right\} .
\end{aligned}
$$


Proof. A standard approximation result for piecewise Hermite bicubic interpolation (see, for example, [5]) gives

$$
\max _{0 \leq n \leq J}\left\|u^{n}-u_{\mathscr{P}}^{n}\right\|_{H^{1}(\Omega)} \leq C h^{3}\|u\|_{C\left([0, T], H^{4}(\Omega)\right)} .
$$

Let $v^{n}=u_{h}^{n}-u_{\mathscr{H}}^{n}, n=0, \ldots, J$. Then $v^{n} \in \mathscr{M}^{0}, v^{0}=0$, and (1.1), (3.1), and (3.7) show that $v^{n}$ satisfies (3.15) with $w^{n}(\xi)=T_{u}^{n}(\xi)$. Hence the required error bound follows from (3.8), (3.17), (3.33) and the triangle inequality.

3.2. ADI Euler scheme. Perturbing the LM Euler scheme (3.1) by an appropriate term that is first-order accurate in time, we obtain the ADI Euler scheme, in which the approximate solution $u_{h}^{n} \in \mathscr{M}, n=1, \ldots, M$, is such that, for $n=0, \ldots, J-1$,

$$
\left[\left(1-\tau \lambda \Delta+\tau^{2} \lambda^{2} \frac{\partial^{4}}{\partial x^{2} \partial y^{2}}\right) \frac{u_{h}^{n+1}-u_{h}^{n}}{\tau}+L^{n} u_{h}^{n}\right](\xi)=f\left(\xi, t_{n}\right), \quad \xi \in \mathscr{G} .
$$

As in the LM Euler scheme, $u_{h}^{0} \in \mathscr{M}$ and $\left.u_{h}^{n}\right|_{\partial \Omega}, n=1, \ldots, J$, are assumed to be given.

The truncation error $T_{u}^{n}(\xi)$ of (3.34) is defined by

$$
T_{u}^{n}(\xi)=\left[\left(\frac{\partial u}{\partial t}\right)^{n}-\left(1-\tau \lambda \Delta+\tau^{2} \lambda^{2} \frac{\partial^{4}}{\partial x^{2} \partial y^{2}}\right) \frac{u_{\mathscr{C}}^{n+1}-u_{\mathscr{C}}^{n}}{\tau}+L^{n}\left(u^{n}-u_{\mathscr{C}}^{n}\right)\right](\xi) .
$$

The following lemma is a counterpart of Lemma 3.1.

Lemma 3.3. Assume $a_{i}, b_{i}, i=1,2$, and c satisfy the assumptions of Lemma 3.1. If $u \in C\left([0, T], H^{6}(\Omega)\right) \cap C^{2,0,1}(\bar{Q}) \cap C^{0,2,1}(\bar{Q}) \cap C^{0,0,2}(\bar{Q})$ and $\partial u / \partial t \in C\left([0, T], H^{3}(\Omega)\right)$, then

$$
\begin{aligned}
& \tau \sum_{n=0}^{J-1}\left\|T_{u}^{n}\right\|_{\mathscr{G}}^{2} \leq C\left\{\tau ^ { 2 } \left[\|u\|_{C^{0,0,2}(\bar{Q})}^{2}\right.\right. \\
& \left.+\lambda^{2}\left(\|u\|_{C^{2,0,1}(\bar{Q})}^{2}+\|u\|_{C^{0,2,1}(\bar{Q})}^{2}\right)+\lambda^{4}\|u\|_{C\left([0, T], H^{6}(\Omega)\right)}^{2}\right] \\
& \left.+h^{6}\left[\left\|\frac{\partial u}{\partial t}\right\|_{C\left([0, T], H^{3}(\Omega)\right)}^{2}+\left(1+\lambda^{2}\right)\|u\|_{C\left([0, T], H^{5}(\Omega)\right)}^{2}\right]\right\} \text {. }
\end{aligned}
$$

Proof. Employing the approach used in the proof of Lemma 4.2 in [2], we can show that

$$
\left\|\frac{\partial^{4}\left(u^{n}-u_{\mathscr{C}}^{n}\right)}{\partial x^{2} \partial y^{2}}\right\|_{\mathscr{G}} \leq C\left\|u^{n}\right\|_{H^{6}(\Omega)} .
$$

The triangle inequality and the Sobolev embedding theorem yield

$$
\left\|\frac{\partial^{4}\left(u_{\mathscr{X}}^{n+1}-u_{\mathscr{C}}^{n}\right)}{\partial x^{2} \partial y^{2}}\right\|_{\mathscr{G}} \leq C\|u\|_{C\left([0, T], H^{6}(\Omega)\right)},
$$

and hence (3.36) follows easily from (3.8). 
Next we show that for $\lambda$ sufficiently large, the ADI Euler scheme (3.34) is unconditionally stable with respect to the initial condition and the right-hand side.

Lemma 3.4. Assume $a_{i}, b_{i}, i=1,2$, and $c$ satisfy the assumptions of Lemma 3.2. If $v^{n}, w^{n} \in \mathscr{M}^{0}$ are such that, for $n=0,1, \ldots, J-1$,

$$
\left[\left(1-\tau \lambda \Delta+\tau^{2} \lambda^{2} \frac{\partial^{4}}{\partial x^{2} \partial y^{2}}\right) \frac{v^{n+1}-v^{n}}{\tau}+L^{n} v^{n}\right](\xi)=w^{n}(\xi), \quad \xi \in \mathscr{G},
$$

where $\lambda$ satisfies (3.16), then (3.17) obtains.

Proof. Let $L_{h}^{n}, \Delta_{h}$ be the operators from $\mathscr{M}^{0}$ into $\mathscr{M}^{0}$ given by (3.18), and let $D_{x x}^{h}, D_{y y}^{h}$ be the operators from $\mathscr{M}^{0}$ into $\mathscr{M}^{0}$ defined by

$$
\left(D_{x x}^{h} v\right)(\xi)=\frac{\partial^{2} v}{\partial x^{2}}(\xi), \quad\left(D_{y y}^{h} v\right)(\xi)=\frac{\partial^{2} v}{\partial y^{2}}(\xi), \quad \xi \in \mathscr{G} .
$$

Taking $v(x, y)=v_{1}(x) v_{2}(y), v_{i} \in \mathscr{M}_{i}^{0}, i=1,2$, we easily verify that

$$
\left(D_{x x}^{h} D_{y y}^{h} v\right)(\xi)=\frac{\partial^{4} v}{\partial x^{2} \partial y^{2}}(\xi), \quad \xi \in \mathscr{G}, \quad v \in \mathscr{M}^{0} .
$$

Therefore, the operator form of (3.38) is given by (2.1), where

$$
A=L_{h}^{n}, \quad B=E-\tau \lambda \Delta_{h}+\tau^{2} \lambda^{2} D_{x x}^{h} D_{y y}^{h} .
$$

It is easy to show that $D_{x x}^{h}=\left[D_{x x}^{h}\right]^{*} \leq 0, D_{y y}^{h}=\left[D_{y y}^{h}\right]^{*} \leq 0$ with respect to $(\cdot, \cdot)_{\mathscr{G}}$, and that $D_{x x}^{h} D_{y y}^{h}=D_{y y}^{h} D_{x x}^{h}$. Thus $D_{x x}^{h} D_{y y}^{h} \geq 0$, and hence (3.17) follows from Theorem 2.1, using (3.27)-(3.32).

Combining the truncation error and stability results, we arrive at the following convergence result for the ADI Euler scheme.

Theorem 3.2. Assume that $a_{i}, b_{i}, i=1,2$, and $c$ satisfy the assumptions of Lemma 3.2 and that the solution $u$ of (1.1) satisfies the assumptions of Lemma 3.3. Let $\lambda$ satisfy (3.16), and let $u_{h}^{n} \in \mathscr{M}, n=1, \ldots, J$, be solutions of (3.34), where $u_{h}^{0} \in \mathscr{M}$ and $\left.u_{h}^{n}\right|_{\partial \Omega}, n=1, \ldots, J$, are given by (3.2), (3.3), respectively. Then

$$
\begin{aligned}
& \max _{0 \leq n \leq J}\left\|u^{n}-u_{h}^{n}\right\|_{H^{1}(\Omega)} \leq C\left\{\tau \left[\|u\|_{C^{0,0,2}(\bar{Q})}+\|u\|_{C^{2,0,1}(\bar{Q})}\right.\right. \\
& \left.+\|u\|_{C^{0,2,1}(\bar{Q})}+\|u\|_{C\left([0, T], H^{6}(\Omega)\right)}\right] \\
& \left.+h^{3}\left[\left\|\frac{\partial u}{\partial t}\right\|_{C\left([0, T], H^{3}(\Omega)\right)}+\|u\|_{C\left([0, T], H^{5}(\Omega)\right)}\right]\right\} \text {. }
\end{aligned}
$$

Proof. The proof of the theorem is similar to that of Theorem 3.1 and follows from (3.36), (3.17) and (3.33).

3.3. ADI Crank-Nicolson scheme. In this subsection, we consider a secondorder in time two-level ADI scheme for the solution of (1.1) with $L=L_{1}+L_{2}$, 
where

(3.40) $L_{1} u=-\frac{\partial}{\partial x}\left(a_{1}(x, y, t) \frac{\partial u}{\partial x}\right), \quad L_{2} u=-\frac{\partial}{\partial y}\left(a_{2}(x, y, t) \frac{\partial u}{\partial y}\right)$.

Let $L_{i}^{n}$ and $L_{i}^{n+1 / 2}, i=1,2$, be differential operators given by (3.40) with $t=$ $t_{n}$ and $t=t_{n+1 / 2} \equiv(n+1 / 2) \tau$, respectively. The ADI Crank-Nicolson scheme consists of finding $u_{h}^{n} \in \mathscr{M}, n=1, \ldots, J$, such that for $n=0, \ldots, J-1$,

$$
\left[\frac{u_{h}^{n+1 / 2}-u_{h}^{n}}{0.5 \tau}+L_{1}^{n+1 / 2} u_{h}^{n+1 / 2}+L_{2}^{n} u_{h}^{n}\right](\xi)=f\left(\xi, t_{n+1 / 2}\right), \quad \xi \in \mathscr{G},
$$

$$
\left[\frac{u_{h}^{n+1}-u_{h}^{n+1 / 2}}{0.5 \tau}+L_{1}^{n+1 / 2} u_{h}^{n+1 / 2}+L_{2}^{n+1} u_{h}^{n+1}\right](\xi)=f\left(\xi, t_{n+1 / 2}\right), \quad \xi \in \mathscr{G},
$$

where $u_{h}^{0} \in \mathscr{M},\left.u_{h}^{n}\right|_{\partial \Omega}, n=1, \ldots, J$, are assumed to be given, and where for each $\xi^{y} \in \mathscr{G}_{y}$, we have $u_{h}^{n+1 / 2}\left(\cdot, \xi^{y}\right) \in \mathscr{M}_{x}$ and

$$
\begin{aligned}
u_{h}^{n+1 / 2}\left(\alpha, \xi^{y}\right)= & {\left[(1 / 2)\left(u_{h}^{n+1}+u_{h}^{n}\right)\right.} \\
& \left.+(\tau / 4)\left(L_{2}^{n+1} u_{h}^{n+1}-L_{2}^{n} u_{h}^{n}\right)\right]\left(\alpha, \xi^{y}\right), \quad \alpha=0,1 .
\end{aligned}
$$

Our convergence analysis of the scheme (3.41) follows that of [26] for the finite difference ADI Crank-Nicolson method. For $\xi \in \mathscr{G}$, the truncation errors $T_{u, 1}^{n}(\xi)$ and $T_{u, 2}^{n}(\xi)$, corresponding respectively to the first and second equations in (3.41), are defined by

$$
\begin{aligned}
T_{u, 1}^{n}(\xi)= & {\left[\left(\frac{\partial u}{\partial t}\right)^{n+1 / 2}-\frac{u_{\mathscr{H}}^{n+1 / 2}-u_{\mathscr{H}}^{n}}{0.5 \tau}\right.} \\
& \left.+\left(L_{1}^{n+1 / 2}+L_{2}^{n+1 / 2}\right) u^{n+1 / 2}-L_{1}^{n+1 / 2} u_{\mathscr{H}}^{n+1 / 2}-L_{2}^{n} u_{\mathscr{H}}^{n}\right](\xi), \\
T_{u, 2}^{n}(\xi)= & {\left[\left(\frac{\partial u}{\partial t}\right)^{n+1 / 2}-\frac{u_{\mathscr{H}}^{n+1}-u_{\mathscr{H}}^{n+1 / 2}}{0.5 \tau}\right.} \\
& \left.+\left(L_{1}^{n+1 / 2}+L_{2}^{n+1 / 2}\right) u^{n+1 / 2}-L_{1}^{n+1 / 2} u_{\mathscr{H}}^{n+1 / 2}-L_{2}^{n+1} u_{\mathscr{H}}^{n+1}\right](\xi),
\end{aligned}
$$

where $u_{\mathscr{H}}^{n}$ is the piecewise Hermite bicubic interpolant of $u\left(\cdot, t_{n}\right)$. For each $\xi^{y} \in \mathscr{G}_{y}$, we define $u_{\mathscr{H}}^{n+1 / 2}\left(\cdot, \xi^{y}\right) \in \mathscr{M}_{x}$ by (cf. $(3.42)$ )

$$
u_{\mathscr{H}}^{n+1 / 2}\left(x, \xi^{y}\right)=\left[(1 / 2)\left(u_{\mathscr{H}}^{n+1}+u_{\mathscr{H}}^{n}\right)+(\tau / 4)\left(z^{n+1}-z^{n}\right)\right]\left(x, \xi^{y}\right),
$$


where $z^{n}\left(\cdot, \xi^{y}\right) \in \mathscr{M}_{x}$ is the piecewise cubic approximation to $L_{2}^{n} u^{n}\left(\cdot, \xi^{y}\right)$ such that

$$
\begin{aligned}
& z^{n}\left(\alpha, \xi^{y}\right)=L_{2}^{n} u_{\mathscr{C}}^{n}\left(\alpha, \xi^{y}\right), \quad \alpha=0,1, \\
& z^{n}\left(x_{k}, \xi^{y}\right)=L_{2}^{n} u^{n}\left(x_{k}, \xi^{y}\right), \quad k=1, \ldots, N_{x}-1, \\
& \frac{\partial z^{n}}{\partial x}\left(x_{k}, \xi^{y}\right)=\frac{\partial L_{2}^{n} u^{n}}{\partial x}\left(x_{k}, \xi^{y}\right), \quad k=0, \ldots, N_{x} .
\end{aligned}
$$

The following lemma gives a bound on the discrete norm of the truncation errors $T_{u, 1}^{n}$ and $T_{u, 2}^{n}$.

Lemma 3.5. Assume $a_{1} \in C^{1,0,0}(\bar{Q})$ and $a_{2} \in C^{0,1,2}(\bar{Q}) \cap C^{2,1,1}(\bar{Q})$. If $u \in$ $C\left([0, T], H^{5}(\Omega)\right) \cap C^{2,2,1}(\bar{Q}) \cap C^{2,0,2}(\bar{Q}) \cap C^{0,2,2}(\bar{Q}) \cap C^{0,0,3}(\bar{Q})$, $L_{2} u \in C^{5,0,0}(\bar{Q}), \quad \partial u / \partial t \in C\left([0, T], H^{3}(\Omega)\right)$, and $\partial^{6} u(\alpha, \cdot, \cdot) / \partial y^{5} \partial t \in$ $C([0,1] \times[0, T]), \quad \alpha=0,1$, then

$$
\begin{aligned}
\tau \sum_{n=0}^{J-1}\left[\left\|T_{u, 1}^{n}\right\|_{\mathscr{G}}^{2}+\left\|T_{u, 2}^{n}\right\|_{\mathscr{Q}}^{2}\right] & \\
\leq C & \left\{\tau^{4}\left[\|u\|_{C^{2,2,1}(\bar{Q})}^{2}+\|u\|_{C^{2,0,2}(\bar{Q})}^{2}+\|u\|_{C^{0,2,2}(\bar{Q})}^{2}+\|u\|_{C^{0,0,3}(\bar{Q})}^{2}\right]\right. \\
& +h^{6}\left[\|u\|_{C\left([0, T], H^{5}(\Omega)\right)}^{2}+\left\|L_{2} u\right\|_{C^{s, 0,0}(\bar{Q})}^{2}+\left\|\frac{\partial u}{\partial t}\right\|_{C\left([0, T], H^{3}(\Omega)\right)}^{2}\right. \\
& \left.\left.+\max _{\alpha=0,1, k=0,1}\left\|\frac{\partial^{5+k} u}{\partial y^{5} \partial t^{k}}(\alpha, \cdot, \cdot)\right\|_{C([0,1] \times[0, T])}^{2}\right]\right\} .
\end{aligned}
$$

Proof. It follows from (3.43)-(3.45) that

$$
\begin{aligned}
& T_{u, 1}^{n}(\xi)=I_{n}^{1}(\xi)+I_{n}^{2}(\xi)+I_{n}^{3}(\xi)-(\tau / 4) I_{n}^{5}(\xi), \\
& T_{u, 2}^{n}(\xi)=I_{n}^{1}(\xi)+I_{n}^{2}(\xi)+I_{n}^{4}(\xi)-(\tau / 4) I_{n}^{5}(\xi),
\end{aligned}
$$

where

$$
\begin{aligned}
I_{n}^{1}(\xi) & =\left(\frac{\partial u}{\partial t}\right)^{n+1 / 2}(\xi)-\frac{u_{\mathscr{X}}^{n+1}-u_{\mathscr{K}}^{n}}{\tau}(\xi), \\
I_{n}^{2}(\xi) & =L_{1}^{n+1 / 2} u^{n+1 / 2}(\xi)-(1 / 2) L_{1}^{n+1 / 2}\left[u_{\mathscr{C}}^{n+1}+u_{\mathscr{C}}^{n}\right](\xi), \\
I_{n}^{3}(\xi) & =L_{2}^{n+1 / 2} u^{n+1 / 2}(\xi)-L_{2}^{n} u_{\mathscr{C}}^{n}(\xi)-(1 / 2)\left(z^{n+1}-z^{n}\right)(\xi), \\
I_{n}^{4}(\xi) & =L_{2}^{n+1 / 2} u^{n+1 / 2}(\xi)-L_{2}^{n+1} u_{\mathscr{K}}^{n+1}(\xi)+(1 / 2)\left(z^{n+1}-z^{n}\right)(\xi), \\
I_{n}^{5}(\xi) & =L_{1}^{n+1 / 2}\left(z^{n+1}-z^{n}\right)(\xi),
\end{aligned}
$$

and where $z^{n}\left(\cdot, \xi^{y}\right) \in \mathscr{M}_{x}$ is defined in (3.46). By Taylor's theorem,

$$
\left|\left(\frac{\partial u}{\partial t}\right)^{n+1 / 2}(\xi)-\frac{u^{n+1}-u^{n}}{\tau}(\xi)\right| \leq \frac{\tau^{2}}{24}\|u\|_{C^{0,0,3}(\bar{\Omega})},
$$


and hence (3.13) and the triangle inequality give

$$
\left\|I_{n}^{1}\right\|_{\mathscr{G}}^{2} \leq C\left[\tau^{4}\|u\|_{C^{0,0,3}(\bar{Q})}^{2}+h^{6}\left\|\frac{\partial u}{\partial t}\right\|_{C\left([0, T], H^{3}(\Omega)\right)}^{2}\right] .
$$

Since

$$
\begin{aligned}
I_{n}^{2}(\xi)= & L_{1}^{n+1 / 2} u^{n+1 / 2}(\xi)-(1 / 2) L_{1}^{n+1 / 2}\left(u^{n+1}+u^{n}\right)(\xi) \\
& +(1 / 2) L_{1}^{n+1 / 2}\left(u^{n+1}-u_{\mathscr{H}}^{n+1}+u^{n}-u_{\mathscr{H}}^{n}\right)(\xi),
\end{aligned}
$$

using Taylor's theorem and (3.9), we obtain

$$
\left\|I_{n}^{2}\right\|_{\mathscr{G}}^{2} \leq C\left[\tau^{4}\|u\|_{C^{2,0,2}(\bar{Q})}^{2}+h^{6}\|u\|_{C\left([0, T], H^{5}(\Omega)\right)}^{2}\right] .
$$

Clearly,

where

$$
I_{n}^{3}(\xi)=J_{n}^{1}(\xi)+J_{n}^{2}(\xi)-(1 / 2) J_{n}^{3}(\xi)+(1 / 2) J_{n+1}^{3},
$$

$$
\begin{aligned}
& J_{n}^{1}(\xi)=L_{2}^{n+1 / 2} u^{n+1 / 2}(\xi)-(1 / 2)\left[L_{2}^{n+1} u^{n+1}+L_{2}^{n} u^{n}\right](\xi), \\
& J_{n}^{2}(\xi)=L_{2}^{n} u^{n}(\xi)-L_{2}^{n} u_{\mathscr{H}}^{n}(\xi), \quad J_{n}^{3}(\xi)=L_{2}^{n} u^{n}(\xi)-z^{n}(\xi) .
\end{aligned}
$$

Using Taylor's theorem and (3.9), we obtain

$$
\left|J_{n}^{1}(\xi)\right| \leq C \tau^{2}\|u\|_{C^{0,2,2}(\bar{Q})}, \quad\left|J_{n}^{2}(\xi)\right| \leq C h^{3}\|u\|_{C\left([0, T], H^{5}(\Omega)\right)} .
$$

For $\xi=\left(\xi^{x}, \xi^{y}\right)$, where $\xi^{x} \in\left[x_{1}, x_{N_{x}-1}\right]$, using (2.17) of [5] with $m=2$, $p=3$, and $q=r=\infty$, we find that

$$
\left|J_{n}^{3}(\xi)\right| \leq C h^{3}\left\|L_{2} u\right\|_{C^{3,0,0}(\bar{Q})} .
$$

To bound $J_{n}^{3}(\xi)$ for $\xi^{x} \notin\left[x_{1}, x_{N_{x}-1}\right]$, we use the following result, which follows from (9.4) and (9.5) of [19] with $r=3, p=1$ and $\alpha=2$ : if $v \in C^{5}[0,1]$ and $v_{\mathscr{H}} \in \mathscr{M}_{x}$ is its piecewise Hermite cubic interpolant, then

$$
\left|\left(v-v_{\mathscr{L}}\right)^{\prime \prime}\left(\xi_{x}\right)\right| \leq C h^{3}\left\|v^{(5)}\right\|_{C[0,1]}, \quad \xi_{x} \in \mathscr{G}_{x} .
$$

Now, if $\xi^{x} \in\left[0, x_{1}\right] \cup\left[x_{N_{x}-1}, 1\right]$, using (3.50), we can show that

$$
\left|J_{n}^{3}(\xi)\right| \leq C h^{3}\left[\left\|L_{2} u\right\|_{C^{3,0,0}(\bar{Q})}+\max _{\alpha=0,1}\left\|\frac{\partial^{5} u}{\partial y^{5}}(\alpha, \cdot, \cdot)\right\|_{C([0,1] \times[0, T])}\right] .
$$

Similar considerations apply also to $I_{n}^{4}(\xi)$. Therefore, for $i=3,4$,

$$
\begin{aligned}
\left\|I_{n}^{i}\right\|_{\mathscr{G}}^{2} \leq C\left\{\tau^{4}\|u\|_{C^{0,2,2}(\bar{Q})}^{2}+h^{6}\right. & {\left[\|u\|_{C\left([0, T], H^{5}(\Omega)\right)}^{2}+\left\|L_{2} u\right\|_{C^{3,0,0}(\bar{Q})}^{2}\right.} \\
& \left.\left.+\max _{\alpha=0,1}\left\|\frac{\partial^{5} u}{\partial y^{5}}(\alpha, \cdot, \cdot)\right\|_{C([0,1] \times[0, T])}^{2}\right]\right\} .
\end{aligned}
$$

Clearly,

$$
I_{n}^{5}(\xi)=J_{n}^{4}(\xi)+J_{n}^{5}(\xi)-J_{n}^{6}(\xi)
$$


where

$$
\begin{gathered}
J_{n}^{4}(\xi)=L_{1}^{n+1 / 2}\left[L_{2}^{n+1} u^{n+1}-L_{2}^{n} u^{n}\right](\xi), \\
J_{n}^{5}(\xi)=L_{1}^{n+1 / 2}\left[L_{2}^{n} u^{n}-z^{n}\right](\xi), \quad J_{n}^{6}(\xi)=L_{1}^{n+1 / 2}\left[L_{2}^{n+1} u^{n+1}-z^{n+1}\right](\xi) .
\end{gathered}
$$

It follows easily from Taylor's theorem that

$$
\left|J_{n}^{4}(\xi)\right| \leq C \tau\|u\|_{C^{2,2,1}(\bar{Q})} .
$$

For $\xi=\left(\xi^{x}, \xi^{y}\right)$ with $\xi^{x} \in\left[x_{1}, x_{N_{x}-1}\right]$, using (3.50), we obtain

$$
\left|J_{n}^{5}(\xi)\right|,\left|J_{n}^{6}(\xi)\right| \leq C h^{3}\left\|L_{2} u\right\|_{C^{5,0,0}(\bar{Q})} \text {. }
$$

If $\xi^{x} \in\left[0, x_{1}\right] \cup\left[x_{N_{x}-1}, 1\right]$, then by (2.5) of [19] it can be shown that

$$
\left|J_{n}^{6}(\xi)-J_{n}^{5}(\xi)\right| \leq C\left[h^{3}\left\|L_{2} u\right\|_{C^{5,0,0}(\bar{Q})}+h \tau \max _{\alpha=0,1}\left\|\frac{\partial^{6} u}{\partial y^{5} \partial t}(\alpha, \cdot, \cdot)\right\|_{C([0,1] \times[0, T])}\right] \text {. }
$$

Therefore,

$$
\begin{aligned}
\left\|I_{n}^{5}\right\|_{\mathscr{G}}^{2} \leq C\{ & h^{6}\left\|L_{2} u\right\|_{C^{5,0,0}(\bar{Q})}^{2} \\
& \left.\quad+\tau^{2}\left[\|u\|_{C^{2,2,1}(\bar{\Omega})}^{2}+\max _{\alpha=0,1}\left\|\frac{\partial^{6} u}{\partial y^{5} \partial t}(\alpha, \cdot, \cdot)\right\|^{2}\right]\right\} .
\end{aligned}
$$

Finally, (3.47) follows from (3.48)-(3.52).

The next lemma shows that, for $h$ sufficiently small, the scheme $(3.41)$ is stable with respect to the initial condition and the right-hand side.

Lemma 3.6. Assume that $a_{1}$ and $a_{2}$ satisfy the assumptions of Lemma 3.2. Let $v^{n}, w_{1}^{n}, w_{2}^{n} \in \mathscr{M}^{0}$ be such that, for $n=0, \ldots, J-1$,

$$
\left[\frac{v^{n+1 / 2}-v^{n}}{0.5 \tau}+L_{1}^{n+1 / 2} v^{n+1 / 2}+L_{2}^{n} v^{n}\right](\xi)=w_{1}^{n}(\xi), \quad \xi \in \mathscr{G}
$$

$$
\left[\frac{v^{n+1}-v^{n+1 / 2}}{0.5 \tau}+L_{1}^{n+1 / 2} v^{n+1 / 2}+L_{2}^{n+1} v^{n+1}\right](\xi)=w_{2}^{n}(\xi), \quad \xi \in \mathscr{G}
$$

where, for each $\xi^{y} \in \mathscr{G}_{y}$, one has $v^{n+1 / 2}\left(\cdot, \xi^{y}\right) \in \mathscr{M}_{x}^{0}$. Then, for $h$ sufficiently small,

$$
\begin{aligned}
\max _{0 \leq n \leq J} & {\left[\left\|v^{n}\right\|_{L^{2}(\Omega)}^{2}+\tau \sum_{k=1}^{N_{x}} \frac{h_{k}^{x}}{2} \sum_{i=1}^{2}\left\|\frac{\partial v^{n}}{\partial y}\left(\xi_{k, i}^{x}, \cdot\right)\right\|_{L^{2}(0,1)}^{2}\right] } \\
& \leq C\left[\left\|\left(1+0.5 \tau L_{2}^{0}\right) v^{0}\right\|_{\mathscr{G}}^{2}+T \frac{\tau}{2} \sum_{n=0}^{J-1}\left(\left\|w_{1}^{n}\right\|_{\mathscr{G}}^{2}+\left\|w_{2}^{n}\right\|_{\mathscr{G}}^{2}\right)\right] .
\end{aligned}
$$


Proof. It follows from (3.53) and the triangle inequality that

$$
\begin{aligned}
& \left\|\left(1+0.5 \tau L_{1}^{n+1 / 2}\right) v^{n+1 / 2}\right\|_{\mathscr{G}} \leq\left\|\left(1-0.5 \tau L_{2}^{n}\right) v^{n}\right\|_{\mathscr{G}}+0.5 \tau\left\|w_{1}^{n}\right\|_{\mathscr{G}}, \\
& \left\|\left(1+0.5 \tau L_{2}^{n+1}\right) v^{n+1}\right\|_{\mathscr{S}} \leq\left\|\left(1-0.5 \tau L_{1}^{n+1 / 2}\right) v^{n+1 / 2}\right\|_{\mathscr{G}}+0.5 \tau\left\|w_{2}^{n}\right\|_{\mathscr{G}} .
\end{aligned}
$$

If $h$ is sufficiently small, then inequality (4.14) of [2] implies that

$$
\left(L_{1}^{n+1 / 2} v, v\right)_{\mathscr{G}} \geq 0, \quad v \in \mathscr{K}_{x}^{0}, \quad\left(L_{2}^{n} v, v\right)_{\mathscr{G}} \geq 0, \quad v \in \mathscr{M}^{0} .
$$

Since

$$
\left\|\left(1 \pm 0.5 \tau L_{1}^{n+1 / 2}\right) v\right\|_{\mathscr{G}}^{2}=\|v\|_{\mathscr{G}}^{2} \pm \tau\left(L_{1}^{n+1 / 2} v, v\right)_{\mathscr{G}}+\frac{\tau^{2}}{4}\left\|L_{1}^{n+1 / 2} v\right\|_{\mathscr{G}}^{2},
$$

the first inequality in (3.56) gives

$$
\left\|\left(1-0.5 \tau L_{1}^{n+1 / 2}\right) v\right\|_{\mathscr{G}} \leq\left\|\left(1+0.5 \tau L_{1}^{n+1 / 2}\right) v\right\|_{\mathscr{G}}, \quad v \in \mathscr{M}_{x}^{0} .
$$

By a similar argument,

$$
\left\|\left(1-0.5 \tau L_{2}^{n}\right) v\right\|_{\mathscr{G}} \leq\left\|\left(1+0.5 \tau L_{2}^{n}\right) v\right\|_{\mathscr{G}}, \quad v \in \mathscr{M}^{0} .
$$

Hence, (3.55), (3.57), and (3.58) yield

$$
\left\|\left(1+0.5 \tau L_{2}^{n+1}\right) v^{n+1}\right\|_{\mathscr{S}} \leq\left\|\left(1+0.5 \tau L_{2}^{n}\right) v^{n}\right\|_{\mathscr{G}}+0.5 \tau\left(\left\|w_{1}^{n}\right\|_{\mathscr{G}}+\left\|w_{2}^{n}\right\|_{\mathscr{G}}\right),
$$

and therefore, for $n=0, \ldots, J$,

$$
\begin{aligned}
\left\|\left(1+0.5 \tau L_{2}^{n}\right) v^{n}\right\|_{\mathscr{G}} \leq & \left\|\left(1+0.5 \tau L_{2}^{0}\right) v^{0}\right\|_{\mathscr{G}} \\
& +0.5 \tau \sum_{n=0}^{J-1}\left(\left\|w_{1}^{n}\right\|_{\mathscr{G}}+\left\|w_{2}^{n}\right\|_{\mathscr{G}}\right) .
\end{aligned}
$$

For $h$ sufficiently small, (2.6) of [25], (4.14) of [2], and (3.2) of [19] give

$$
\begin{aligned}
& \left\|\left(1+0.5 \tau L_{2}^{n}\right) v^{n}\right\|_{\mathscr{G}}^{2} \\
& \quad \geq C\left[\left\|v^{n}\right\|_{L^{2}(\bar{\Omega})}^{2}+\tau \sum_{k=1}^{N_{x}} \frac{h_{k}^{x}}{2} \sum_{i=1}^{2}\left\|\frac{\partial v^{n}}{\partial y}\left(\xi_{k, i}^{x}, \cdot\right)\right\|_{L^{2}(0,1)}^{2}\right] .
\end{aligned}
$$

Hence, (3.54) follows from (3.59) and (3.60).

Finally we arrive at the following convergence result.

Theorem 3.3. Assume that $a_{1}, a_{2}$ satisfy the assumptions of Lemmas 3.5 and 3.6. Let $u$ be the solution of (1.1) satisfying the assumptions of Lemma 3.5. Let $u_{h}^{n} \in \mathscr{M}, n=1, \ldots, J$, be solutions of (3.41), where $u_{h}^{0} \in \mathscr{M}$ and $\left.u_{h}^{n}\right|_{\partial \Omega}$, $n=1, \ldots, J$, are given by (3.2) and (3.3), respectively. Then, for $h$ sufficiently 
small,

$$
\begin{gathered}
\max _{0 \leq n \leq J}\left[\left\|u^{n}-u_{h}^{n}\right\|_{L^{2}(\Omega)}^{2}+\tau \sum_{k=1}^{N_{x}} \frac{h_{k}^{x}}{2} \sum_{i=1}^{2}\left\|\frac{\partial\left(u^{n}-u_{h}^{n}\right)}{\partial y}\left(\xi_{k, i}^{x}, \cdot\right)\right\|_{L^{2}(0,1)}^{2}\right]^{1 / 2} \\
\leq C\left\{\tau^{2}\left[\|u\|_{C^{2,2,1}(\bar{Q})}+\|u\|_{C^{2,0,2}(\bar{Q})}+\|u\|_{C^{0,2,2}(\bar{Q})}+\|u\|_{C^{0,0,3}(\bar{Q})}\right]\right. \\
+h^{3}\left[\|u\|_{C\left([0, T], H^{5}(\Omega)\right)}+\left\|L_{2} u\right\|_{C^{5,0,0}(\bar{Q})}+\left\|\frac{\partial u}{\partial t}\right\|_{C\left([0, T], H^{3}(\Omega)\right)}\right. \\
\left.\left.+\max _{\alpha=0,1, k=0,1}\left\|\frac{\partial^{5+k} u}{\partial y^{5} \partial t^{k}}(\alpha, \cdot, \cdot)\right\|_{C([0,1] \times[0, T])}\right]\right\} .
\end{gathered}
$$

Proof. Let $v^{n}=u_{h}^{n}-u_{\mathscr{H}}^{n}, n=0, \ldots, J$, and let $v^{n+1 / 2}=u_{h}^{n+1 / 2}-u_{\mathscr{H}}^{n+1 / 2}, n=$ $0, \ldots, J-1$, where $u_{\mathscr{K}}^{n+1 / 2}$ is defined by (3.45). Then (1.1) and (3.41) imply that $v^{n}, v^{n+1 / 2}$ satisfy $(3.53)$ with $w_{1}^{n}(\xi)=T_{u, 1}^{n}(\xi)$ and $w_{2}^{n}(\xi)=T_{u, 2}^{n}(\xi)$, where $T_{u, 1}^{n}(\xi)$ and $T_{u, 2}^{n}(\xi)$ are given by (3.43) and (3.44), respectively. Clearly, $v^{n} \in \mathscr{M}^{0}, v^{0}=0$, and $v^{n+1 / 2}\left(\cdot, \xi^{y}\right) \in \mathscr{M}_{x}^{0}$. Hence the required inequality follows from (3.54), (3.47), (3.33) and the triangle inequality.

Theorem 3.3 shows that the ADI Crank-Nicolson orthogonal spline collocation approximation $u_{h}^{n}$ converges to the exact solution $u^{n}$ with accuracy $O\left(\tau^{2}+h^{3}\right)$ in a norm that is stronger than the $L^{2}(\Omega)$-norm but weaker than the $H^{1}(\Omega)$-norm.

\section{THREE-LEVEL SCHEMES}

In this section, we present and analyze three three-level piecewise Hermite bicubic orthogonal spline collocation schemes for the approximate solution of the parabolic problem (1.1).

4.1. Laplace-modified method. In the orthogonal spline collocation LM method which is a counterpart of the finite element Galerkin LM method of [18], the approximate solution $u_{h}^{n} \in \mathscr{M}, n=2,3, \ldots, J$, is such that for $n=$ $1, \ldots, J-1$,

$$
\left[\frac{u_{h}^{n+1}-u_{h}^{n-1}}{2 \tau}-\tau^{2} \lambda \Delta \frac{u_{h}^{n+1}-2 u_{h}^{n}+u_{h}^{n-1}}{\tau^{2}}+L^{n} u_{h}^{n}\right](\xi)=f\left(\xi, t_{n}\right), \quad \xi \in \mathscr{G},
$$

where $u_{h}^{0}, u_{h}^{1} \in \mathscr{M}$ and $\left.u_{h}^{n}\right|_{\partial \Omega}, n=2, \ldots, J$, are assumed to be given.

As in the case of the two-level schemes, we use the piecewise Hermite bicubic interpolant of the exact solution as the comparison function. Hence, for $n=$ $1, \ldots, J-1$ and $\xi \in \mathscr{G}$, the truncation error $T_{u}^{n}(\xi)$ of $(4.1)$ is defined by

$$
T_{u}^{n}(\xi)=\left[\left(\frac{\partial u}{\partial t}\right)^{n}-\frac{u_{\mathscr{C}}^{n+1}-u_{\mathscr{H}}^{n-1}}{2 \tau}+\tau^{2} \lambda \Delta \frac{u_{\mathscr{P}}^{n+1}-2 u_{\mathscr{P}}^{n}+u_{\mathscr{P}}^{n-1}}{\tau^{2}}+L^{n}\left(u^{n}-u_{\mathscr{P}}^{n}\right)\right](\xi) .
$$


A bound on the discrete norm of the truncation error is given in the following lemma.

Lemma 4.1. Assume that $a_{i}, b_{i}, i=1,2$, and $c$ satisfy the assumptions of Lemma 3.1. If $u \in C\left([0, T], H^{5}(\Omega)\right) \cap C^{2,0,2}(\bar{Q}) \cap C^{0,2,2}(\bar{Q}) \cap C^{0,0,3}(\bar{Q})$ and $\partial u / \partial t \in C\left([0, T], H^{3}(\Omega)\right)$, then

$$
\begin{aligned}
\tau \sum_{n=1}^{J-1}\left\|T_{u}^{n}\right\|_{\mathscr{G}}^{2} \leq C\{ & \tau^{4}\left[\|u\|_{C^{0,0,3}(\bar{Q})}^{2}+\lambda^{2}\left(\|u\|_{C^{2,0,2}(\bar{Q})}^{2}+\|u\|_{C^{0,2,2}(\bar{Q})}^{2}\right)\right] \\
& \left.+h^{6}\left[\left\|\frac{\partial u}{\partial t}\right\|_{C\left([0, T], H^{3}(\Omega)\right)}^{2}+\left(1+\lambda^{2}\right)\|u\|_{C\left([0, T], H^{5}(\Omega)\right)}^{2}\right]\right\} .
\end{aligned}
$$

Proof. By Taylor's theorem,

$$
\left(\frac{\partial u}{\partial t}\right)^{n}(\xi)-\frac{u^{n+1}-u^{n-1}}{2 \tau}(\xi)=-\frac{\tau^{2}}{6} \frac{\partial^{3} u}{\partial t^{3}}\left(\xi, \tilde{t}_{\xi, n}\right)
$$

and

$$
\Delta\left(u^{n+1}-2 u^{n}+u^{n-1}\right)(\xi)=\tau^{2} \frac{\partial^{2}(\Delta u)}{\partial t^{2}}\left(\xi, \bar{t}_{\xi, n}\right),
$$

where $t_{n-1} \leq \tilde{t}_{\xi, n}, \bar{t}_{\xi, n} \leq t_{n+1}$. Hence, (4.3) follows from the triangle inequality and arguments similar to those used in the proof of Lemma 3.1.

Next we show that if $\lambda$ is sufficiently large, then the LM scheme (4.1) is unconditionally stable with respect to the initial condition and the right-hand side.

Lemma 4.2. Assume $a_{i}, b_{i}, i=1,2$, and c satisfy the assumptions of Lemma 3.2. Let $v^{n}, w^{n} \in \mathscr{M}^{0}$ be such that, for $n=1, \ldots, J-1$,

$$
\left[\frac{v^{n+1}-v^{n-1}}{2 \tau}-\tau^{2} \lambda \Delta \frac{v^{n+1}-2 v^{n}+v^{n-1}}{\tau^{2}}+L^{n} v^{n}\right](\xi)=w^{n}(\xi), \quad \xi \in \mathscr{G}
$$

where

$$
\lambda>a_{\max } / 4 .
$$

Then there exists a positive constant $M$, independent of $h$ and $\tau$, such that

(4.6) $\max _{0 \leq n \leq J}\left\|v^{n}\right\|_{H^{\prime}(\Omega)}^{2} \leq M\left[\left(-\Delta v^{0}, v^{0}\right)_{\mathscr{G}}+\left(-\Delta v^{1}, v^{1}\right)_{\mathscr{G}}+\tau \sum_{n=0}^{J-1}\left\|w^{n}\right\|_{\mathscr{G}}^{2}\right]$.

Proof. Let $L_{h}^{n}$ and $\Delta_{h}$ be the operators from $\mathscr{M}^{0}$ into $\mathscr{M}^{0}$ defined in (3.18). Then the operator form of (4.4) is given by (2.13), where

$$
A^{(n)}=L_{h}^{n}, \quad B=E, \quad R=-\lambda \Delta_{h} .
$$

It follows from (3.27)-(3.31) and (4.5) that all the assumptions of Theorem 2.2 are satisfied for $H=\mathscr{M}^{0}$, with $A_{0}=-\Delta_{h}, \varepsilon_{0}=a_{\min }, \varepsilon_{1}=$ $K \max \left(a_{\min }^{-1},\left[4 \lambda-a_{\max }\right]^{-1}\right), \varepsilon_{2}=C \delta^{2}$, where $\delta$ is defined in (3.25), $\varepsilon_{3}=1$, $\varepsilon_{4}=4 \lambda / a_{\max }-1$. Therefore, (4.6) follows from (2.19) and (3.32). 
The truncation error and stability results yield the following convergence theorem.

Theorem 4.1. Assume that $a_{i}, b_{i}, i=1,2$, and $c$ satisfy the assumptions of Lemma 3.2 and that the solution $u$ of (1.1) satisfies the assumptions of Lemma 4.1. Let $\lambda$ satisfy (4.5), and let $u_{h}^{n} \in \mathscr{M}, n=2, \ldots, J-1$, be solutions of (4.1), where $u_{h}^{0} \in \mathscr{M}$ and $\left.u_{h}^{n}\right|_{\partial \Omega}, n=1, \ldots, J$, are given by (3.2) and (3.3), respectively. Then

$$
\begin{aligned}
\max _{0 \leq n \leq J}\left\|u^{n}-u_{h}^{n}\right\|_{H^{1}(\Omega)} \leq C\{ & \left(-\Delta\left(u_{h}^{1}-u_{\mathscr{C}}^{1}\right), u_{h}^{1}-u_{\mathscr{Z}}^{1}\right)_{\mathscr{G}}^{1 / 2} \\
& +\tau^{2}\left[\|u\|_{C^{0,0,3}(\bar{Q})}+\|u\|_{C^{2,0,2}(\bar{Q})}+\|u\|_{C^{0,2,2}(\bar{Q})}\right] \\
& \left.+h^{3}\left[\left\|\frac{\partial u}{\partial t}\right\|_{C\left([0, T], H^{3}(\Omega)\right)}+\|u\|_{C\left([0, T], H^{5}(\Omega)\right)}\right]\right\} .
\end{aligned}
$$

Proof. Let $v^{n}=u_{h}^{n}-u_{\mathscr{C}}^{n}, n=0, \ldots, J$. Then $v^{n} \in \mathscr{M}^{0}, v^{0}=0$, and (1.1), (4.1) imply that the $v^{n}$ satisfy $(4.4)$ with $w^{n}(\xi)=T_{u}^{n}(\xi)$, where $T_{u}^{n}(\xi)$ is defined by (4.2). Hence the required inequality follows from (4.3) and (4.6).

We now explain how $u_{h}^{1} \in \mathscr{M}$ can be selected, and we also bound the term

$$
\left(-\Delta\left(u_{h}^{1}-u_{\mathscr{L}}^{1}\right), u_{h}^{1}-u_{\mathscr{H}}^{1}\right)_{\mathscr{G}}^{1 / 2},
$$

which appears on the right-hand side of (4.7). For the first choice of $u_{h}^{1}$, we assume that the differential equation in (1.1) is satisfied for $t=0$. Then, using Taylor's theorem, we obtain

$$
u^{1}(x, y)=z(x, y)+O\left(\tau^{2}\right),
$$

where

$$
z(x, y)=g_{1}(x, y)+\tau\left[f(x, y, 0)-L^{0} g_{1}(x, y)\right] .
$$

As stated in Theorem 4.1, $\left.u_{h}^{1}\right|_{\partial \Omega}$ is given by (3.3) with $n=1$. To complete the definition of $u_{h}^{1}$, we also require that

$$
\begin{aligned}
& \frac{\partial^{i+j}\left(u_{h}^{1}-z\right)}{\partial x^{i} \partial y^{j}}\left(x_{k}, y_{l}\right)=0, \quad i, j=0,1, \quad 1 \leq k \leq N_{x}-1, \quad 1 \leq l \leq N_{y}-1, \\
& \frac{\partial^{i+1}\left(u_{h}^{1}-z\right)}{\partial x^{i} \partial y}\left(x_{k}, \alpha\right)=0, \quad i=0,1, \quad 1 \leq k \leq N_{x}-1, \quad \alpha=0,1, \\
& \frac{\partial^{1+j}\left(u_{h}^{1}-z\right)}{\partial x \partial y^{j}}\left(\alpha, y_{l}\right)=0, \quad j=0,1, \quad 1 \leq l \leq N_{y}-1, \quad \alpha=0,1, \\
& \frac{\partial^{2}\left(u_{h}^{1}-z\right)}{\partial x \partial y}(\alpha, \beta)=0, \quad \alpha, \beta=0,1 .
\end{aligned}
$$

If $z_{\mathscr{H}}$ is the piecewise Hermite bicubic interpolant of $z$, then it is easy to see that $u_{h}^{1}=z_{\mathscr{K}}$ on all interior partition cells, that is, cells $\left[x_{k-1}, x_{k}, y_{l-1}, y_{l}\right]$ which do not have common points with $\partial \Omega$. (The choice $u_{h}^{1}=z_{\mathscr{K}}$ on $\Omega$ would lead, in general, to $u_{h}^{1}-u_{\mathscr{P}}^{1} \neq 0$ on $\partial \Omega$, which would prevent us from 
using Lemma 4.2 in the proof of Theorem 4.1.) To bound (4.8), we use the obvious identity

$$
v \equiv u_{h}^{1}-u_{\mathscr{H}}^{1}=v_{1}-v_{2}-v_{3}+v_{4}
$$

where

$$
v_{1}=u^{1}-u_{\mathscr{H}}^{1}, \quad v_{2}=z-z_{\mathscr{H}}, \quad v_{3}=u^{1}-z, \quad v_{4}=u_{h}^{1}-z_{\mathscr{H}} .
$$

Let $\mathscr{G}^{*}$ be the set of all Gauss points in $\mathscr{G}$ which are not in the interior partition cells. Assuming that $u$ and $z$ are sufficiently smooth, using (3.9), Taylor's theorem and Theorem 5 of [5], we can show that

$$
\begin{gathered}
\left\|\Delta v_{1}\right\|_{\mathscr{G}},\left\|\Delta v_{2}\right\|_{\mathscr{G}} \leq C h^{3}, \quad\left\|\Delta v_{3}\right\|_{\mathscr{G}} \leq C \tau^{2}, \\
\left|\Delta v_{4}(\xi)\right| \leq C \tau^{2} h^{-2}, \quad \xi \in \mathscr{G}^{*},
\end{gathered}
$$

(4.12) $\left|v_{1}(\xi)\right|,\left|v_{2}(\xi)\right| \leq C h^{4}, \quad \xi \in \mathscr{G}, \quad\left|v_{3}(\xi)\right|,\left|v_{4}(\xi)\right| \leq C \tau^{2}, \quad \xi \in \mathscr{G}$

Therefore, it follows from the Cauchy-Schwarz inequality that

$$
\left|(\Delta v, v)_{\mathscr{G}}\right| \leq \sum_{i=1}^{3} \sum_{j=1}^{4}\left\|\Delta v_{i}\right\|_{\mathscr{G}}\left\|v_{j}\right\|_{\mathscr{G}}+h^{2} \sum_{\xi \in \mathscr{G}^{*}}\left|\Delta v_{4}(\xi)\right||v(\xi)| \leq C\left(\tau^{4} h^{-1}+h^{6}\right),
$$

and hence

$$
\left(-\Delta\left(u_{h}^{1}-u_{\mathscr{H}}^{1}\right), u_{h}^{1}-u_{\mathscr{H}}^{1}\right)_{\mathscr{G}}^{1 / 2} \leq C\left(\tau^{2} h^{-1 / 2}+h^{3}\right)
$$

It should be noted that if $\left(\partial^{2} u / \partial t^{2}\right)(x, y, t)=0$ for $(x, y) \in \partial \Omega$, which for example, occurs when $g_{2}$ is independent of $t$, then $h^{-1 / 2}$ does not appear in (4.13).

Another way of choosing $u_{h}^{1} \in \mathscr{M}$, where $\left.u_{h}^{1}\right|_{\partial \Omega}$ is given as before, is to perform one step of the Crank-Nicolson scheme,

$$
\left[\frac{u_{h}^{1}-u_{h}^{0}}{\tau}+\frac{1}{2}\left(L^{1} u_{h}^{1}+L^{0} u_{h}^{0}\right)\right](\xi)=\frac{1}{2}\left[f\left(\xi, t_{1}\right)+f\left(\xi, t_{0}\right)\right], \quad \xi \in \mathscr{G} .
$$

Obviously, computing $u_{h}^{1}$ in this case requires the solution of one elliptic orthogonal spline collocation problem. To bound (4.8), we set $v^{n}=u_{h}^{n}-u_{\mathscr{H}}^{n}$, $n=0,1$. Then $v^{0}=0, v^{1} \in \mathscr{M}^{0}$, and (1.1), (4.14) yield

$$
\left[\left(2 E+\tau A^{(1)}\right) v^{1}\right](\xi)=2 \tau w(\xi), \quad \xi \in \mathscr{G},
$$

where $A^{(1)}=L_{h}^{1}$ is an operator from $\mathscr{M}^{0}$ into $\mathscr{M}^{0}$ defined by (3.18) with $n=1$, and $w \in \mathscr{M}^{0}$ is given by

$$
w(\xi)=\frac{1}{2} \sum_{n=0}^{1}\left(\frac{\partial u}{\partial t}\right)^{n}(\xi)-\frac{u_{\mathscr{X}}^{1}-u_{\mathscr{X}}^{0}}{\tau}(\xi)+\frac{1}{2} \sum_{n=0}^{1} L^{n}\left(u^{n}-u_{\mathscr{H}}^{n}\right)(\xi), \quad \xi \in \mathscr{G} .
$$


If $u$ is sufficiently smooth, then it is easy to show that

$$
\|w\|_{\mathscr{S}} \leq C\left(\tau^{2}+h^{3}\right) .
$$

Now, using (3.27) with $n=1$ in (4.15), and then taking the inner product $(\cdot, \cdot)_{\mathscr{G}}$ of both sides with $v^{1}$, we obtain

$$
2\left\|v^{1}\right\|_{\mathscr{G}}^{2}+\tau\left(A_{0}^{(1)} v^{1}, v^{1}\right)_{\mathscr{G}}=2 \tau\left(w, v^{1}\right)_{\mathscr{G}}-\tau\left(A_{1}^{(1)} v^{1}, v^{1}\right)_{\mathscr{G}} .
$$

Using the Cauchy-Schwarz inequality, the inequality

$$
\alpha \beta \leq(3 / 8) \tau \alpha^{2}+(2 / 3) \tau^{-1} \beta^{2},
$$

and (3.30), we obtain

$$
\begin{aligned}
& 2 \tau\left(w, v^{1}\right)_{\mathscr{G}}-\tau\left(A_{1}^{(1)} v^{1}, v^{1}\right)_{\mathscr{G}} \\
& \quad \leq 2\left\|v^{1}\right\|_{\mathscr{G}}^{2}+\frac{3}{4} \tau^{2}\|w\|_{\mathscr{G}}^{2}+\frac{3}{8} C \delta^{2} \tau^{2}\left(-\Delta_{h} v^{1}, v^{1}\right)_{\mathscr{G}} .
\end{aligned}
$$

Thus, it follows from (4.17), (4.18), (3.28), and (4.16) that for $\tau$ sufficiently small,

$$
\left(-\Delta\left(u_{h}^{1}-u_{\mathscr{P}}^{1}\right), u_{h}^{1}-u_{\mathscr{H}}^{1}\right)_{\mathscr{G}}^{1 / 2} \leq C \tau^{1 / 2}\|w\|_{\mathscr{G}} \leq C \tau^{1 / 2}\left(\tau^{2}+h^{3}\right) .
$$

4.2. ADI methods. In this subsection, we present two three-level ADI schemes which are obtained by perturbing the LM scheme (4.1). The schemes of this section are orthogonal spline collocation counterparts of the corresponding finite element Galerkin schemes proposed in $[15,18]$. In the first three-level ADI method, the approximate solution $u_{h}^{n} \in \mathscr{M}, n=2,3, \ldots, J$, satisfies

$$
\begin{aligned}
& {\left[\left(1+4 \tau^{2} \lambda^{2} \frac{\partial^{4}}{\partial x^{2} \partial y^{2}}\right) \frac{u_{h}^{n+1}-u_{h}^{n-1}}{2 \tau}\right.} \\
& \left.-\tau^{2} \lambda \Delta \frac{u_{h}^{n+1}-2 u_{h}^{n}+u_{h}^{n-1}}{\tau^{2}}+L^{n} u_{h}^{n}\right](\xi)=f\left(\xi, t_{n}\right), \\
& \xi \in \mathscr{G}, \quad n=1, \ldots, J-1,
\end{aligned}
$$

where $u_{h}^{0}, u_{h}^{1} \in \mathscr{M}$ and $\left.u_{h}^{n}\right|_{\partial \Omega}, n=1, \ldots, J$, are assumed to be given.

For $n=1, \ldots, J-1$ and $\xi \in \mathscr{G}$, the truncation error $T_{u}^{n}(\xi)$ of the scheme (4.19) is defined by

$$
\begin{aligned}
T_{u}^{n}(\xi)=\left[\left(\frac{\partial u}{\partial t}\right)^{n}\right. & -\left(1+4 \tau^{2} \lambda^{2} \frac{\partial^{4}}{\partial x^{2} \partial y^{2}}\right) \frac{u_{\mathscr{H}}^{n+1}-u_{\mathscr{H}}^{n-1}}{2 \tau} \\
& \left.+\tau^{2} \lambda \Delta \frac{u_{\mathscr{K}}^{n+1}-2 u_{\mathscr{K}}^{n}+u_{\mathscr{H}}^{n-1}}{\tau^{2}}+L^{n}\left(u^{n}-u_{\mathscr{X}}^{n}\right)\right](\xi) .
\end{aligned}
$$

Lemma 4.3. Assume that $a_{i}, b_{i}, i=1,2$, and $c$ satisfy the assumptions of Lemma 3.1. If $u \in C\left([0, T], H^{5}(\Omega)\right) \cap C^{2,0,2}(\bar{Q}) \cap C^{0,2,2}(\bar{Q}) \cap C^{0,0,3}(\bar{Q})$ 
and $\partial u / \partial t \in C\left([0, T], H^{6}(\Omega)\right)$, then

$$
\begin{aligned}
\tau \sum_{n=1}^{J-1}\left\|T_{u}^{n}\right\|_{\mathscr{G}}^{2} \leq C\left\{\tau ^ { 4 } \left[\|u\|_{C^{0,0,3}(\bar{Q})}^{2}\right.\right. & \\
& \left.+\lambda^{2}\left(\|u\|_{C^{2,0,2}(\bar{Q})}^{2}+\|u\|_{C^{0,2,2}(\bar{Q})}^{2}\right)+\lambda^{4}\left\|\frac{\partial u}{\partial t}\right\|_{C\left([0, T], H^{6}(\Omega)\right)}^{2}\right] \\
& \left.+h^{6}\left[\left\|\frac{\partial u}{\partial t}\right\|_{C\left([0, T], H^{3}(\Omega)\right)}^{2}+\left(1+\lambda^{2}\right)\|u\|_{C\left([0, T], H^{5}(\Omega)\right)}^{2}\right]\right\} .
\end{aligned}
$$

Proof. Since $\partial u_{\mathscr{L}} / \partial t=(\partial u / \partial t)_{\mathscr{H}}$, following (3.13) and using (3.37), we obtain

$$
\begin{aligned}
& \left\|\frac{\partial^{4}}{\partial x^{2} \partial y^{2}}\left(\frac{u_{\mathscr{H}}^{n+1}-u_{\mathscr{H}}^{n-1}}{2 \tau}\right)-\frac{\partial^{4}}{\partial x^{2} \partial y^{2}}\left(\frac{u^{n+1}-u^{n-1}}{2 \tau}\right)\right\|_{\mathscr{G}}^{2} \\
& \quad=\left\|\frac{1}{2 \tau} \int_{(n-1) \tau}^{(n+1) \tau} \frac{\partial^{4}}{\partial x^{2} \partial y^{2}} \frac{\partial}{\partial t}\left(u_{\mathscr{H}}-u\right)(\cdot, s) d s\right\|_{\mathscr{G}}^{2} \\
& \quad \leq \frac{1}{2 \tau} \int_{(n-1) \tau}^{(n+1) \tau}\left\|\frac{\partial^{4}}{\partial x^{2} \partial y^{2}}\left[\left(\frac{\partial u}{\partial t}\right)_{\mathscr{H}}-\frac{\partial u}{\partial t}\right](\cdot, s)\right\|_{\mathscr{G}}^{2} d s \\
& \quad \leq C\left\|\frac{\partial u}{\partial t}\right\|_{C\left([0, T], H^{6}(\Omega)\right)}^{2} \cdot
\end{aligned}
$$

By a similar argument and the Sobolev embedding theorem,

$$
\begin{gathered}
\left\|\frac{\partial^{4}}{\partial x^{2} \partial y^{2}}\left(\frac{u^{n+1}-u^{n-1}}{2 \tau}\right)\right\|_{\mathscr{G}}^{2}=\left\|\frac{1}{2 \tau} \int_{(n-1) \tau}^{(n+1) \tau} \frac{\partial^{4}}{\partial x^{2} \partial y^{2}} \frac{\partial u}{\partial t}(\cdot, s) d s\right\|_{\mathscr{G}}^{2} \\
\leq \frac{1}{2 \tau} \int_{(n-1) \tau}^{(n+1) \tau}\left\|\frac{\partial^{4}}{\partial x^{2} \partial y^{2}} \frac{\partial u}{\partial t}(\cdot, s)\right\|_{\mathscr{G}}^{2} d s \leq C\left\|\frac{\partial u}{\partial t}\right\|_{C\left([0, T], H^{6}(\Omega)\right)}^{2}
\end{gathered} .
$$

Hence, the triangle inequality, (4.22) and (4.23) give

$$
\left\|\frac{\partial^{4}}{\partial x^{2} \partial y^{2}}\left(\frac{u_{\mathscr{H}}^{n+1}-u_{\mathscr{H}}^{n-1}}{2 \tau}\right)\right\|_{\mathscr{G}} \leq C\left\|\frac{\partial u}{\partial t}\right\|_{C\left([0, T], H^{6}(\Omega)\right)} .
$$

Inequality (4.21) now follows from (4.3) and (4.24).

The convergence result for the scheme (4.19) is given in the following theorem.

Theorem 4.2. Assume that $a_{i}, b_{i}, i=1,2$, and $c$ satisfy the assumptions of Lemma 3.2 and that the solution $u$ of (1.1) satisfies the assumptions of Lemma 4.3. Let $\lambda$ satisfy (4.5), and let $u_{h}^{n} \in \mathscr{M}, n=2, \ldots, J-1$, be the solution of (4.19), where $u_{h}^{0} \in \mathscr{M}$ and $\left.u_{h}^{n}\right|_{\partial \Omega}, n=1, \ldots, J$, are given by (3.2) and 
(3.3), respectively. Then

$$
\begin{aligned}
\max _{0 \leq n \leq J}\left\|u^{n}-u_{h}^{n}\right\|_{H^{1}(\Omega)} & \\
\leq C\{( & \left.-\Delta\left(u_{h}^{1}-u_{\mathscr{C}}^{1}\right), u_{h}^{1}-u_{\mathscr{Z}}^{1}\right)_{\mathscr{G}}^{1 / 2} \\
& +\tau^{2}\left[\|u\|_{C^{0,0,3}(\bar{Q})}+\|u\|_{C^{2,0,2}(\bar{Q})}+\|u\|_{C^{0,2,2}(\bar{Q})}+\left\|\frac{\partial u}{\partial t}\right\|_{C\left([0, T], H^{6}(\Omega)\right)}\right] \\
& \left.+h^{3}\left[\left\|\frac{\partial u}{\partial t}\right\|_{C\left([0, T], H^{3}(\Omega)\right)}+\|u\|_{C\left([0, T], H^{5}(\Omega)\right)}\right]\right\} .
\end{aligned}
$$

Proof. Let $L_{h}^{n}, \Delta_{h}$ and $D_{x x}^{h}, D_{y y}^{h}$ be the operators from $\mathscr{M}^{0}$ into $\mathscr{M}^{0}$ defined in (3.18) and (3.39), respectively. Let $w^{n} \in \mathscr{M}^{0}$ be such that $w^{n}(\xi)=T_{u}^{n}(\xi)$, $\xi \in \mathscr{G}$, where $T_{u}^{n}(\xi)$ is given by (4.20). Then (1.1) and (4.19) imply that the $v^{n}=u_{h}^{n}-u_{\mathscr{C}}^{n} \in \mathscr{M}^{0}$ satisfy (2.13) with

$$
A^{(n)}=L_{h}^{n}, \quad B=E+4 \tau^{2} \lambda^{2} D_{x x}^{h} D_{y y}^{h}, \quad R=-\lambda \Delta_{h} .
$$

Since $D_{x x}^{h} D_{y y}^{h} \geq 0$ (see the proof of Lemma 3.4), it follows from the results established in the proof of Lemma 4.2 that all assumptions of Theorem 2.2 are satisfied. Therefore, the desired inequality is a consequence of (2.19), (4.21) and (3.32).

As in the three-level LM method, $u_{h}^{1}$ can be selected again in one of the two ways which are described at the end of $\S 4.1$.

In the second three-level ADI scheme, the approximate solution $u_{h}^{n} \in \mathscr{M}$, $n=2,3, \ldots, J$, satisfies

$$
\begin{aligned}
& {\left[\frac{u_{h}^{n+1}-u_{h}^{n-1}}{2 \tau}\right.} \\
& \left.-\left(\tau^{2} \lambda \Delta-2 \tau^{3} \lambda^{2} \frac{\partial^{4}}{\partial x^{2} \partial y^{2}}\right) \frac{u_{h}^{n+1}-2 u_{h}^{n}+u_{h}^{n-1}}{\tau^{2}}+L^{n} u_{h}^{n}\right](\xi) \\
& \quad=f\left(\xi, t_{n}\right), \quad \xi \in \mathscr{G}, \quad n=1, \ldots, J-1,
\end{aligned}
$$

where $u_{h}^{0}, u_{h}^{1}$, and $\left.u_{h}^{n}\right|_{\partial \Omega}, n=2, \ldots, J$, are assumed to be given.

The truncation error $T_{u}^{n}(\xi), \quad n=1, \ldots, J-1, \xi \in \mathscr{G}$, of the scheme (4.25) is defined by

$$
\begin{aligned}
T_{u}^{n}(\xi)=\left[\left(\frac{\partial u}{\partial t}\right)^{n}-\frac{u_{\mathscr{X}}^{n+1}-u_{\mathscr{H}}^{n-1}}{2 \tau}+\left(\tau^{2} \lambda \Delta-2 \tau^{3} \lambda^{2} \frac{\partial^{4}}{\partial x^{2} \partial y^{2}}\right)\right. \\
\left.\cdot \frac{u_{\mathscr{X}}^{n+1}-2 u_{\mathscr{H}}^{n}+u_{\mathscr{H}}^{n-1}}{\tau^{2}}+L^{n}\left(u^{n}-u_{\mathscr{C}}^{n}\right)\right](\xi) .
\end{aligned}
$$

Lemma 4.4. Assume $a_{i}, b_{i}, i=1,2, c$, and $u$ satisfy the assumptions of Lemma 4.3. Then $T_{u}^{n}$ given by (4.26) satisfies (4.21). 
Proof. Writing $u_{\mathscr{H}}^{n+1}-2 u_{\mathscr{H}}^{n}+u_{\mathscr{H}}^{n-1}$ as $\left(u_{\mathscr{H}}^{n+1}-u_{\mathscr{H}}^{n}\right)-\left(u_{\mathscr{H}}^{n}-u_{\mathscr{H}}^{n-1}\right)$ and using arguments similar to those in $(4.22)-(4.24)$, we can easily show that

$$
\left\|\frac{\partial^{4}}{\partial x^{2} \partial y^{2}}\left(\frac{u_{\mathscr{H}}^{n+1}-2 u_{\mathscr{H}}^{n}+u_{\mathscr{H}}^{n-1}}{\tau}\right)\right\|_{\mathscr{G}} \leq C\left\|\frac{\partial u}{\partial t}\right\|_{C\left([0, T], H^{6}(\Omega)\right)} .
$$

Hence, inequality (4.21) follows from (4.3) and (4.27).

The convergence result for the scheme (4.25) is given in the following theorem.

Theorem 4.3. Assume that $a_{i}, b_{i}, i=1,2, c, u$, and $\lambda$ satisfy the assumptions of Theorem 4.2. Let $u_{h}^{n} \in \mathscr{M}, n=2, \ldots, J-1$, be solutions of (4.25), where $u_{h}^{0} \in \mathscr{M}$ and $\left.u_{h}^{n}\right|_{\partial \Omega}, n=1, \ldots, J$, are given by (3.2) and (3.3), respectively. Then

$$
\begin{aligned}
\max _{0 \leq n \leq J} \| u^{n}- & u_{h}^{n} \|_{H^{1}(\Omega)} \\
\leq C & \left\{\left(-\Delta\left(u_{h}^{1}-u_{\mathscr{H}}^{1}\right),\left(u_{h}^{1}-u_{\mathscr{H}}^{1}\right)\right)_{\mathscr{G}}^{1 / 2}\right. \\
& +\tau\left(\frac{\partial^{2}}{\partial x^{2}}\left(u_{h}^{1}-u_{\mathscr{H}}^{1}\right), \frac{\partial^{2}}{\partial y^{2}}\left(u_{h}^{1}-u_{\mathscr{L}}^{1}\right)\right)_{\mathscr{G}}^{1 / 2} \\
& +\tau^{2}\left[\|u\|_{C^{0,0,3}(\bar{Q})}+\|u\|_{C^{2,0,2}(\bar{Q})}+\|u\|_{C^{0,2,2}(\bar{Q})}+\left\|\frac{\partial u}{\partial t}\right\|_{C\left([0, T], H^{6}(\Omega)\right)}\right] \\
& \left.+h^{3}\left[\left\|\frac{\partial u}{\partial t}\right\|_{C\left([0, T], H^{3}(\Omega)\right)}+\|u\|_{C\left([0, T], H^{5}(\Omega)\right)}\right]\right\}
\end{aligned}
$$

Proof. The proof of the desired inequality follows from the results established in the proofs of Lemmas 4.2, 3.4, Lemma 4.4, and Theorem 2.2 applied to (2.13) with the operators

$$
A^{(n)}=L_{h}^{n}, \quad B=E, \quad R=-\lambda \Delta_{h}+2 \tau \lambda^{2} D_{x x}^{h} D_{y y}^{h} .
$$

As in three-level LM method, $u_{h}^{1}$ can be chosen as in (4.9), so that if $u$ and $z$ are sufficiently smooth, then (4.13) is satisfied. Moreover, using (4.10)-(4.12), we can also show that

$$
\left|\tau\left(\frac{\partial^{2}\left(u_{h}^{1}-u_{\mathscr{H}}^{1}\right)}{\partial x^{2}}, \frac{\partial^{2}\left(u_{h}^{1}-u_{\mathscr{H}}^{1}\right)}{\partial y^{2}}\right)_{\mathscr{G}}^{1 / 2}\right| \leq C\left(\tau^{3} h^{-3 / 2}+h^{3}\right) .
$$

It should be pointed out again that if $\left(\partial^{2} u / \partial t^{2}\right)(x, y, t)=0$ for $(x, y) \in \partial \Omega$, which is the case when $g_{2}$ is independent of $t$, then $h^{-3 / 2}$ does not appear in the last inequality.

\section{ACKNOWLEDGEMENTS}

Bernard Bialecki was supported in part by the National Science Foundation grant CCR-9103451. Ryan I. Fernandes was supported by the Center for Computational Sciences and the Center for Applied Energy Research at the University of Kentucky. 


\section{BIBLIOGRAPHY}

1. V. K. Bangia, C. Bennett, A. Reynolds, R. Raghavan, and G. Thomas, Alternating direction collocation methods for simulating reservoir performance, Paper SPE 7414, 53rd SPE Fall Technical Conference and Exhibition, Houston, Texas, 1978.

2. B. Bialecki and X. C. Cai, $H^{1}$-error bounds for $C^{1}$ piecewise Hermite bicubic orthogonal spline collocation schemes, submitted.

3. B. Bialecki, G. Fairweather, and K. R. Bennett, Fast direct solvers for piecewise Hermite bicubic orthogonal spline collocation equations, SIAM J. Numer. Anal. 29 (1992), 156-173.

4. B. Bialecki and R. I. Fernandes, Implementation of orthogonal spline collocation Laplacemodified and alternating-direction methods for parabolic problems, in preparation.

5. G. Birkhoff, M. H. Schultz, and R. S. Varga, Piecewise Hermite interpolation in one and two variables with applications to partial differential equations, Numer. Math. 11 (1968), 232-256.

6. J. F. Botha and M. Celia, The alternating direction collocation approximation, Proceedings of the Eighth South African Symposium on Numerical Mathematics, Durban, South Africa, July, 1982, pp. 13-26.

7. M. A. Celia, L. R. Ahuja, and G. F. Pinder, Orthogonal collocation and alternating-direction methods for unsaturated flow, Adv. Water Resources 10 (1987), 178-187.

8. M. A. Celia and G. F. Pinder, Collocation solution of the transport equation using a locally enhanced alternating direction formulation, Unification of Finite Element Methods $(\mathrm{H}$. Kardestuncer, ed.), Elsevier Science Publishers, New York, 1984, pp. 303-320.

9. __ An analysis of alternating-direction collocation methods for parabolic equations, Numer. Methods Partial Differential Equations 1 (1985), 57-70.

10. Generalized alternating-direction collocation methods for parabolic equations. I. Spatially varying coefficients, Numer. Methods Partial Differential Equations 6 (1990), 193214.

11. - Generalized alternating-direction collocation methods for parabolic equations. II. Transport equations with application to seawater intrusion problems, Numer. Methods Partial Differential Equations 6 (1990), 215-230.

12. __ Generalized alternating-direction collocation methods for parabolic equations. III. Nonrectangular domains, Numer. Methods Partial Differential Equations 6 (1990), 231243.

13. M. A. Celia, G. F. Pinder, and L. J. Hayes, Alternating-direction collocation solution to the transport equation, Proc. Third Internat. Conf. Finite Elements in Water Resources (S. Y. Wang et al., eds.), Univ. of Mississippi, Oxford, MS, 1980, pp. 3.36-3.48.

14. J. H. Cerutti and S. V. Parter, Collocation methods for parabolic partial differential equations in one space dimension, Numer. Math. 26 (1976), 227-254.

15. J. E. Dendy, An analysis of some Galerkin schemes for the solution of nonlinear timedependent problems, SIAM J. Numer. Anal. 28 (1975), 541-565.

16. J. C. Diaz, G. Fairweather, and P. Keast, FORTRAN packages for solving certain almost block diagonal linear systems by modified alternate row and column elimination, ACM Trans. Math. Software 9 (1983), 358-375.

17. _ Algorithm 603-COLROW and ARCECO: FORTRAN packages for solving certain almost block diagonal linear systems by modified alternate row and column elimination, ACM Trans. Math. Software 9 (1983), 376-380.

18. J. Douglas, Jr. and T. Dupont, Alternating direction Galerkin methods on rectangles, Numerical Solution of Partial Differential Equations-II (B. Hubbard, ed.), Academic Press, New York, 1971, pp. 133-214.

19. Collocation methods for parabolic equations in a single space variable, Lecture Notes in Math., vol. 385, Springer-Verlag, New York, 1974.

20. R. I. Fernandes, Alternating direction finite element methods for solving time dependent problems, Ph. D. Thesis, University of Kentucky, Lexington, KY, 1991. 
21. R. I. Fernandes and G. Fairweather, Analysis of alternating direction collocation methods for parabolic and hyperbolic problems in two space variables, Numer. Methods Partial Differential Equations 9 (1993), (to appear).

22. L. J. Hayes, An alternating-direction collocation method for finite element approximations on rectangles, Comput. Math. Appl. 6 (1980), 45-50.

23. _ A comparison of alternating-direction collocation methods for the transport equation, New Concepts in Finite Element Analysis (T. J. R. Hughes et al., eds.), AMS-Vol. 44, American Society of Mechanical Engineers, New York, 1981, pp. 169-177.

24. M. Lees, A priori estimates for the solutions of difference approximations to parabolic partial differential equations, Duke Math. J. 27 (1960), 297-311.

25. P. Percell and M. F. Wheeler, $A C^{1}$ finite element collocation method for elliptic equations, SIAM J. Numer. Anal. 17 (1980), 605-622.

26. A. A. Samarskii, Theory of difference schemes, "Nauka”, Moscow, 1977 (Russian).

27. A. A. Samarskii and A. B. Gulin, Stability of difference schemes, "Nauka", Moscow, 1973 (Russian).

Department of Mathematics, University of Kentucky, LeXington, Kentucky 40506 E-mail address, B. Bialecki: bialecki@ms.uky.edu

E-mail address, R. I. Fernandes: super062@ukcc.uky.edu 